Supplement of Atmos. Meas. Tech., 12, 6647-6665, 2019

https://doi.org/10.5194/amt-12-6647-2019-supplement

(C) Author(s) 2019. This work is distributed under

the Creative Commons Attribution 4.0 License.

(c) (i)

\author{
Atmospheric \\ Measurement \\ Techniques
}

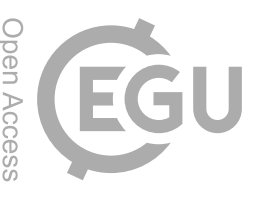

Supplement of

\title{
Field comparison of dry deposition samplers for collection of atmospheric mineral dust: results from single-particle characterization
}

\author{
Andebo Waza et al. \\ Correspondence to: Andebo Waza (andebo.waza@geo.tu-darmstadt.de)
}

The copyright of individual parts of the supplement might differ from the CC BY 4.0 License. 


\section{Measurement}

Table S 1: A daily basis mass deposition rate $\left(\mathbf{m g} /\left(\mathbf{m}^{2} \mathbf{d}\right)\right)$ shown in each size interval measured by Vertical deposition samplers.

$\mathrm{FP}=$ Flat plate, $\mathrm{Sig}=$ Sigma-2

\begin{tabular}{|c|c|c|c|c|c|c|c|c|c|}
\hline \multirow{3}{*}{$\begin{array}{l}\text { Samp. } \\
\text { Id. }\end{array}$} & \multirow{3}{*}{ Date } & \multirow{3}{*}{$\begin{array}{c}\text { Temp } \\
\left({ }^{\circ} \mathrm{C}\right)\end{array}$} & \multirow{3}{*}{$\begin{array}{l}\text { Wind } \\
\text { speed } \\
(\mathrm{m} / \mathrm{s})\end{array}$} & \multicolumn{6}{|c|}{ Mass deposition rate $\left(\mathrm{mg} /\left(\mathrm{m}^{2} \mathrm{~d}\right)\right)$} \\
\hline & & & & \multicolumn{6}{|c|}{ Size interval (aerodynamic diameter) } \\
\hline & & & & $1-2 \mu \mathrm{m}$ & $2-4 \mu \mathrm{m}$ & $4-8 \mu \mathrm{m}$ & $8-16 \mu \mathrm{m}$ & $\begin{array}{c}16-32 \\
\mu \mathrm{m}\end{array}$ & $\begin{array}{c}32-64 \\
\mu \mathrm{m}\end{array}$ \\
\hline FP & 02.08 .17 & 15.997 & 2.055 & 0.0125 & 0.0332 & 0.1591 & 0.6915 & 1.0487 & 0 \\
\hline FP & 03.08 .17 & 16.832 & 2.182 & 0.0178 & 0.085 & 0.4457 & 3.1786 & 3.5723 & 12.2381 \\
\hline FP & 09.08 .17 & 22.064 & 3.045 & 0.2318 & 2.5612 & 12.1382 & 22.3694 & 17.5472 & 0 \\
\hline FP & 10.08 .17 & 22.041 & 2.694 & 0.1268 & 1.7105 & 7.6181 & 14.3374 & 10.8418 & 7.2271 \\
\hline FP & 20.08 .17 & 23.049 & 2.321 & 0.0587 & 0.9965 & 7.7644 & 31.8469 & 39.2924 & 0 \\
\hline FP & 21.08 .17 & 22.127 & 1.991 & 0.0625 & 1.2487 & 9.9323 & 29.9751 & 22.0631 & 0 \\
\hline FP & 22.08 .17 & 21.126 & 3.011 & 0.0413 & 1.0977 & 8.4911 & 16.1487 & 9.563 & 0 \\
\hline FP & 16.07.17 & 21.407 & 1.687 & 0.1512 & 1.6673 & 5.8333 & 10.3739 & 6.789 & 0 \\
\hline FP & 18.07.17 & 19.863 & 3.289 & 0.0773 & 1.0835 & 4.3797 & 5.3431 & 4.4326 & 0 \\
\hline FP & 19.07.17 & 17.691 & 4.275 & 0.0191 & 0.2874 & 1.4257 & 2.5807 & 0.7248 & 0 \\
\hline FP & 20.07.17 & 15.915 & 4.620 & 0.0084 & 0.0437 & 0.3936 & 1.7336 & 2.0418 & 0 \\
\hline FP & 24.07 .17 & 20.758 & 3.883 & 0.1656 & 2.1236 & 8.9983 & 13.6345 & 2.7224 & 0 \\
\hline FP & 25.07 .17 & 20.784 & 2.055 & 0.1468 & 1.5641 & 6.7421 & 11.8483 & 0.7455 & 0 \\
\hline FP & 26.07 .17 & 20.989 & 2.550 & 0.0819 & 1.0551 & 5.0441 & 7.0303 & 1.8159 & 0 \\
\hline FP & 27.07 .17 & 21.563 & 2.092 & 0.1272 & 1.3325 & 6.6209 & 5.5919 & 0.8894 & 0 \\
\hline FP & 28.07.17 & 22.325 & 2.318 & 0.0912 & 1.2328 & 4.7233 & 4.0095 & 1.9389 & 0 \\
\hline FP & 29.07 .17 & 21.971 & 4.438 & 0.0145 & 0.0798 & 0.5085 & 0.7329 & 1.9062 & 0 \\
\hline FP & 30.07 .17 & 19.823 & 4.874 & 0.08 & 0.2918 & 1.2003 & 1.9713 & 0 & 0 \\
\hline Sig & 02.08 .17 & 15.997 & 2.055 & 0.0133 & 0.0435 & 0.3232 & 1.4541 & 1.1561 & 0 \\
\hline $\mathrm{Sig}$ & 03.08 .17 & 16.832 & 2.182 & 0.0117 & 0.0637 & 0.2629 & 0.7752 & 0.7638 & 0 \\
\hline $\mathrm{Sig}$ & 04.08 .17 & 18.734 & 3.251 & 0.0537 & 0.2111 & 0.6742 & 0.9907 & 0.2664 & 0 \\
\hline $\mathrm{Sig}$ & 09.08 .17 & 22.064 & 3.045 & 0.2747 & 3.2314 & 12.3996 & 21.4241 & 7.4674 & 0 \\
\hline $\mathrm{Sig}$ & 10.08 .17 & 22.041 & 2.694 & 0.1171 & 1.312 & 4.7166 & 7.9581 & 1.4412 & 0 \\
\hline $\mathrm{Sig}$ & 11.08 .17 & 20.230 & 4.490 & 0.0506 & 0.3731 & 1.4438 & 3.2957 & 3.822 & 5.9832 \\
\hline $\mathrm{Sig}$ & 12.08 .17 & 18.666 & 4.551 & 0.0435 & 0.3212 & 1.0996 & 2.4052 & 2.1606 & 0 \\
\hline Sig & 14.08 .17 & 19.269 & 5.154 & 0.0929 & 0.6751 & 2.6105 & 5.3232 & 6.6811 & 0 \\
\hline Sig & 15.08 .17 & 19.918 & 1.855 & 0.0113 & 0.0581 & 0.2661 & 0.384 & 1.164 & 0 \\
\hline $\mathrm{Sig}$ & 16.08 .17 & 22.284 & 1.523 & 0.1003 & 0.973 & 3.507 & 5.4434 & 4.8978 & 0 \\
\hline $\mathrm{Sig}$ & 17.08 .17 & 21.384 & 2.978 & 0.1022 & 0.6704 & 2.382 & 4.9459 & 4.6931 & 0 \\
\hline Sig & 18.08.17 & 20.574 & 3.080 & 0.0423 & 0.2089 & 0.7355 & 1.606 & 1.455 & 0 \\
\hline
\end{tabular}




\begin{tabular}{|l|c|c|c|c|c|c|c|c|c|} 
Sig & 19.08 .17 & 22.836 & 2.388 & 0.0337 & 0.2013 & 0.9165 & 2.0097 & 2.0509 & 0 \\
\hline Sig & 20.08 .17 & 23.049 & 2.321 & 0.2254 & 2.5137 & 12.8442 & 44.6417 & 42.0205 & 11.7559 \\
\hline Sig & 21.08 .17 & 22.127 & 1.991 & 0.3337 & 4.1199 & 20.8917 & 48.5994 & 36.6512 & 6.6514 \\
\hline Sig & 22.08 .17 & 21.126 & 3.011 & 0.2849 & 3.5102 & 16.3368 & 35.5797 & 17.1256 & 0 \\
\hline Sig & 20.07 .17 & 15.915 & 4.620 & 0.0102 & 0.0431 & 0.2539 & 1.8245 & 2.4863 & 0 \\
\hline Sig & 21.07 .17 & 14.153 & 3.830 & 0.0093 & 0.0405 & 0.1077 & 0.5439 & 0 & 0 \\
\hline Sig & 26.07 .17 & 20.989 & 2.550 & 0.1704 & 2.0074 & 8.3447 & 11.0216 & 3.496 & 0 \\
\hline Sig & 27.07 .17 & 21.563 & 2.092 & 0.1692 & 2.3802 & 10.0826 & 9.6501 & 4.2398 & 0 \\
\hline Sig & 28.07 .17 & 22.325 & 2.318 & 0.2126 & 2.3481 & 9.5403 & 10.2896 & 1.9448 & 0 \\
\hline Sig & 29.07 .17 & 21.971 & 4.438 & 0.1927 & 2.1052 & 8.1321 & 8.5173 & 1.0481 & 0 \\
\hline Sig & 30.07 .17 & 19.823 & 4.874 & 0.4671 & 1.2559 & 2.7535 & 4.3127 & 6.5318 & 0 \\
\hline
\end{tabular}

Table S 2: A daily basis number deposition rate $\left(\mathbf{1} /\left(\mathbf{m}^{\mathbf{2}} \mathbf{d}\right)\right)$ shown in each interval measured by Vertical deposition samplers.

$\mathrm{FP}=$ Flat plate, $\mathrm{Sig}=$ Sigma-2

\begin{tabular}{|c|c|c|c|c|c|c|c|c|c|}
\hline \multirow{3}{*}{$\begin{array}{l}\text { Samp } \\
\text {. Id. }\end{array}$} & \multirow{3}{*}{ Date } & \multirow{3}{*}{$\begin{array}{l}\text { Temp } \\
\left({ }^{\circ} \mathrm{C}\right)\end{array}$} & \multirow{3}{*}{$\begin{array}{l}\text { Wind } \\
\text { speed } \\
(\mathrm{m} / \mathrm{s})\end{array}$} & \multicolumn{6}{|c|}{ Number deposition rate $1 /\left(\mathrm{m}^{2} \mathrm{~d}\right)$} \\
\hline & & & & \multicolumn{6}{|c|}{ Size interval (aerodynamic diameter) } \\
\hline & & & & $1-2 \mu \mathrm{m}$ & $2-4 \mu \mathrm{m}$ & $4-8 \mu \mathrm{m}$ & $8-16 \mu \mathrm{m}$ & $16-32 \mu \mathrm{m}$ & $32-64 \mu \mathrm{m}$ \\
\hline FP & 02.08 .17 & 15.997 & 2.055 & $2.64 \mathrm{E}+06$ & $9.95 \mathrm{E}+05$ & $6.99 \mathrm{E}+05$ & $2.69 \mathrm{E}+05$ & $2.70 \mathrm{E}+05$ & 0 \\
\hline FP & 03.08 .17 & 16.832 & 2.182 & $4.22 \mathrm{E}+06$ & $2.39 \mathrm{E}+06$ & $1.43 \mathrm{E}+06$ & $1.14 \mathrm{E}+06$ & $1.99 \mathrm{E}+05$ & $8.50 \mathrm{E}+04$ \\
\hline FP & 09.08 .17 & 22.064 & 3.045 & $4.12 \mathrm{E}+07$ & $6.57 \mathrm{E}+07$ & $4.39 \mathrm{E}+07$ & $1.10 \mathrm{E}+07$ & $8.10 \mathrm{E}+05$ & 0 \\
\hline FP & 10.08 .17 & 22.041 & 2.694 & $2.17 \mathrm{E}+07$ & $4.28 \mathrm{E}+07$ & $2.70 \mathrm{E}+07$ & $6.87 \mathrm{E}+06$ & $6.30 \mathrm{E}+05$ & $4.00 \mathrm{E}+04$ \\
\hline FP & 20.08 .17 & 23.049 & 2.321 & $9.70 \mathrm{E}+06$ & $2.33 \mathrm{E}+07$ & $2.49 \mathrm{E}+07$ & $1.20 \mathrm{E}+07$ & $2.79 \mathrm{E}+06$ & 0 \\
\hline FP & 21.08 .17 & 22.127 & 1.991 & $9.52 \mathrm{E}+06$ & $2.87 \mathrm{E}+07$ & $3.42 \mathrm{E}+07$ & $1.35 \mathrm{E}+07$ & $1.66 \mathrm{E}+06$ & 0 \\
\hline FP & 22.08 .17 & 21.126 & 3.011 & $6.48 \mathrm{E}+06$ & $2.43 \mathrm{E}+07$ & $2.87 \mathrm{E}+07$ & $7.87 \mathrm{E}+06$ & $5.40 \mathrm{E}+05$ & 0 \\
\hline FP & 16.07 .17 & 21.407 & 1.687 & $2.72 \mathrm{E}+07$ & $4.26 \mathrm{E}+07$ & $2.25 \mathrm{E}+07$ & $5.69 \mathrm{E}+06$ & $4.40 \mathrm{E}+05$ & 0 \\
\hline FP & 18.07 .17 & 19.863 & 3.289 & $1.33 \mathrm{E}+07$ & $2.74 \mathrm{E}+07$ & $1.66 \mathrm{E}+07$ & $3.02 \mathrm{E}+06$ & $3.20 \mathrm{E}+05$ & 0 \\
\hline $\mathrm{FP}$ & 19.07 .17 & 17.691 & 4.275 & $3.34 \mathrm{E}+06$ & $6.59 \mathrm{E}+06$ & $4.97 \mathrm{E}+06$ & $1.32 \mathrm{E}+06$ & $8.40 \mathrm{E}+04$ & 0 \\
\hline FP & 20.07 .17 & 15.915 & 4.620 & $1.80 \mathrm{E}+06$ & $1.34 \mathrm{E}+06$ & $1.27 \mathrm{E}+06$ & $6.96 \mathrm{E}+05$ & $7.20 \mathrm{E}+04$ & 0 \\
\hline FP & 24.07 .17 & 20.758 & 3.883 & $2.83 \mathrm{E}+07$ & $5.16 \mathrm{E}+07$ & $3.29 \mathrm{E}+07$ & $7.71 \mathrm{E}+06$ & $2.30 \mathrm{E}+05$ & 0 \\
\hline FP & 25.07 .17 & 20.784 & 2.055 & $2.58 \mathrm{E}+07$ & $4.07 \mathrm{E}+07$ & $2.47 \mathrm{E}+07$ & $6.18 \mathrm{E}+06$ & $5.00 \mathrm{E}+04$ & 0 \\
\hline FP & 26.07 .17 & 20.989 & 2.550 & $4.22 \mathrm{E}+06$ & $1.59 \mathrm{E}+07$ & $1.87 \mathrm{E}+07$ & $5.13 \mathrm{E}+06$ & $3.50 \mathrm{E}+05$ & 0 \\
\hline FP & 27.07 .17 & 21.563 & 2.092 & $2.46 \mathrm{E}+07$ & $3.40 \mathrm{E}+07$ & $2.47 \mathrm{E}+07$ & $3.59 \mathrm{E}+06$ & $1.20 \mathrm{E}+05$ & 0 \\
\hline FP & 28.07 .17 & 22.325 & 2.318 & $1.59 \mathrm{E}+07$ & $3.19 \mathrm{E}+07$ & $1.75 \mathrm{E}+07$ & $2.43 \mathrm{E}+06$ & $8.00 \mathrm{E}+04$ & 0 \\
\hline FP & 29.07 .17 & 21.971 & 4.438 & $2.80 \mathrm{E}+06$ & $2.08 \mathrm{E}+07$ & $1.48 \mathrm{E}+06$ & $4.25 \mathrm{E}+05$ & $1.50 \mathrm{E}+05$ & 0 \\
\hline FP & 30.07 .17 & 19.823 & 4.874 & $1.98 \mathrm{E}+07$ & $7.50 \mathrm{E}+06$ & $4.85 \mathrm{E}+06$ & $9.70 \mathrm{E}+05$ & 0 & 0 \\
\hline Sig & 02.08 .17 & 15.997 & 2.055 & $2.70 \mathrm{E}+06$ & $1.32 \mathrm{E}+06$ & $9.72 \mathrm{E}+05$ & $5.13 \mathrm{E}+05$ & $8.10 \mathrm{E}+04$ & 0 \\
\hline Sig & 03.08 .17 & 16.832 & 2.182 & $2.55 \mathrm{E}+06$ & $1.83 \mathrm{E}+06$ & $9.93 \mathrm{E}+05$ & $2.95 \mathrm{E}+05$ & $5.40 \mathrm{E}+04$ & 0 \\
\hline Sig & 04.08 .17 & 18.734 & 3.251 & $1.12 \mathrm{E}+07$ & $6.50 \mathrm{E}+06$ & $2.69 \mathrm{E}+06$ & $4.80 \mathrm{E}+05$ & $3.00 \mathrm{E}+04$ & 0 \\
\hline Sig & 09.08 .17 & 22.064 & 3.045 & $4.64 \mathrm{E}+07$ & $8.29 \mathrm{E}+07$ & $4.79 \mathrm{E}+07$ & $1.08 \mathrm{E}+07$ & $5.80 \mathrm{E}+05$ & 0 \\
\hline
\end{tabular}




\begin{tabular}{|c|c|c|c|c|c|c|c|c|c|}
\hline Sig & 10.08 .17 & 22.041 & 2.694 & $2.00 \mathrm{E}+07$ & $3.34 \mathrm{E}+07$ & $1.82 \mathrm{E}+07$ & $4.23 \mathrm{E}+06$ & $1.30 \mathrm{E}+05$ & 0 \\
\hline Sig & 11.08 .17 & 20.230 & 4.490 & $9.72 \mathrm{E}+06$ & $1.05 \mathrm{E}+07$ & $5.75 \mathrm{E}+06$ & $1.93 \mathrm{E}+06$ & $3.30 \mathrm{E}+05$ & $4.00 \mathrm{E}+04$ \\
\hline Sig & 12.08 .17 & 18.666 & 4.551 & $8.52 \mathrm{E}+06$ & $8.49 \mathrm{E}+06$ & $3.71 \mathrm{E}+06$ & $1.13 \mathrm{E}+06$ & $1.33 \mathrm{E}+05$ & 0 \\
\hline Sig & 14.08 .17 & 19.269 & 5.154 & $1.82 \mathrm{E}+07$ & $1.77 \mathrm{E}+07$ & $1.01 \mathrm{E}+07$ & $2.65 \mathrm{E}+06$ & $3.50 \mathrm{E}+05$ & 0 \\
\hline Sig & 15.08 .17 & 19.918 & 1.855 & $2.07 \mathrm{E}+06$ & $1.56 \mathrm{E}+06$ & $9.83 \mathrm{E}+05$ & $2.18 \mathrm{E}+05$ & $5.50 \mathrm{E}+04$ & 0 \\
\hline Sig & 16.08 .17 & 22.284 & 1.523 & $1.83 \mathrm{E}+07$ & $2.45 \mathrm{E}+07$ & $1.33 \mathrm{E}+07$ & $2.77 \mathrm{E}+06$ & $2.30 \mathrm{E}+05$ & 0 \\
\hline Sig & 17.08 .17 & 21.384 & 2.978 & $2.10 \mathrm{E}+07$ & $1.85 \mathrm{E}+07$ & $8.83 \mathrm{E}+06$ & $2.45 \mathrm{E}+06$ & $3.60 \mathrm{E}+05$ & 0 \\
\hline Sig & 18.08 .17 & 20.574 & 3.080 & $9.04 \mathrm{E}+06$ & $5.60 \mathrm{E}+06$ & $2.85 \mathrm{E}+06$ & $7.56 \mathrm{E}+05$ & $1.03 \mathrm{E}+05$ & 0 \\
\hline Sig & 19.08 .17 & 22.836 & 2.388 & $6.42 \mathrm{E}+06$ & $5.32 \mathrm{E}+06$ & $3.42 \mathrm{E}+06$ & $9.50 \mathrm{E}+05$ & $1.52 \mathrm{E}+05$ & 0 \\
\hline Sig & 20.08 .17 & 23.049 & 2.321 & $3.69 \mathrm{E}+07$ & $6.64 \mathrm{E}+07$ & $4.57 \mathrm{E}+07$ & $1.98 \mathrm{E}+07$ & $2.63 \mathrm{E}+06$ & $6.00 \mathrm{E}+04$ \\
\hline Sig & 21.08 .17 & 22.127 & 1.991 & $5.52 \mathrm{E}+07$ & $1.04 \mathrm{E}+08$ & $7.10 \mathrm{E}+07$ & $2.22 \mathrm{E}+07$ & $2.50 \mathrm{E}+06$ & $1.00 \mathrm{E}+05$ \\
\hline Sig & 22.08 .17 & 21.126 & 3.011 & $4.74 \mathrm{E}+07$ & $8.96 \mathrm{E}+07$ & $5.80 \mathrm{E}+07$ & $1.73 \mathrm{E}+07$ & $1.45 \mathrm{E}+06$ & 0 \\
\hline Sig & 20.07 .17 & 15.915 & 4.620 & $2.11 \mathrm{E}+06$ & $1.15 \mathrm{E}+06$ & $7.38 \mathrm{E}+05$ & $7.12 \mathrm{E}+05$ & $1.27 \mathrm{E}+05$ & 0 \\
\hline Sig & 21.07 .17 & 14.153 & 3.830 & $2.12 \mathrm{E}+06$ & $1.25 \mathrm{E}+06$ & $4.24 \mathrm{E}+05$ & $2.74 \mathrm{E}+05$ & 0 & 0 \\
\hline Sig & 26.07 .17 & 20.989 & 2.550 & $3.05 \mathrm{E}+07$ & $5.15 \mathrm{E}+07$ & $3.06 \mathrm{E}+07$ & $6.54 \mathrm{E}+06$ & $3.10 \mathrm{E}+05$ & 0 \\
\hline Sig & 27.07 .17 & 21.563 & 2.092 & $2.95 \mathrm{E}+07$ & $5.97 \mathrm{E}+07$ & $3.74 \mathrm{E}+07$ & $5.75 \mathrm{E}+06$ & $4.30 \mathrm{E}+05$ & 0 \\
\hline Sig & 28.07 .17 & 22.325 & 2.318 & $3.58 \mathrm{E}+07$ & $6.28 \mathrm{E}+07$ & $3.54 \mathrm{E}+07$ & $5.81 \mathrm{E}+06$ & $2.00 \mathrm{E}+05$ & 0 \\
\hline Sig & 29.07 .17 & 21.971 & 4.438 & $3.20 \mathrm{E}+07$ & $5.78 \mathrm{E}+07$ & $3.10 \mathrm{E}+07$ & $4.68 \mathrm{E}+06$ & $1.00 \mathrm{E}+05$ & 0 \\
\hline Sig & 30.07 .17 & 19.823 & 4.874 & $1.04 \mathrm{E}+08$ & $4.09 \mathrm{E}+07$ & $9.80 \mathrm{E}+06$ & $2.10 \mathrm{E}+06$ & $3.00 \mathrm{E}+05$ & 0 \\
\hline
\end{tabular}

Table S 3: A daily basis mass deposition rate $\left(\mathbf{m g} /\left(\mathbf{m}^{2} \mathbf{d}\right)\right)$ shown in each interval measured by

Horizontal deposition samplers. Mass deposition rate in each size interval is also shown

$\mathrm{MW}=\mathrm{MWAC}, \mathrm{BS}=\mathrm{BSNE}$

\begin{tabular}{|c|c|c|c|c|c|c|c|c|c|}
\hline \multirow{3}{*}{$\begin{array}{l}\text { Samp. } \\
\text { Id. }\end{array}$} & \multirow{3}{*}{ Date } & \multirow{3}{*}{$\begin{array}{l}\text { Temp. } \\
\left({ }^{\circ} \mathrm{C}\right)\end{array}$} & \multirow{3}{*}{$\begin{array}{l}\text { Wind Speed } \\
(\mathrm{m} / \mathrm{s})\end{array}$} & \multicolumn{6}{|c|}{ Mass deposition rate $\left(\mathbf{m g} /\left(\mathbf{m}^{2} \mathbf{d}\right)\right)$} \\
\hline & & & & \multicolumn{6}{|c|}{ Size interval (aerodynamic diameter) } \\
\hline & & & & $\begin{array}{l}1-2 \\
\mu \mathrm{m}\end{array}$ & $2-4 \mu \mathrm{m}$ & $4-8 \mu \mathrm{m}$ & $\begin{array}{l}8-16 \\
\mu \mathrm{m}\end{array}$ & $\begin{array}{c}16-32 \\
\mu \mathrm{m}\end{array}$ & $32-64 \mu \mathrm{m}$ \\
\hline MW & 02.08 .17 & 15.997 & 2.055 & 0.053 & 0.3195 & 3.5756 & 18.9069 & 12.3191 & 0 \\
\hline MW & 04.08 .17 & 18.734 & 3.251 & 0.0308 & 0.1555 & 0.7479 & 0.9514 & 2.5171 & 0 \\
\hline MW & 08.08 .17 & 22.889 & 4.303 & 0.573 & 4.788 & 63.067 & 431.618 & 596.928 & 18.604 \\
\hline MW & 09.08 .17 & 22.064 & 3.045 & 0.3805 & 3.7223 & 53.2968 & 357.1174 & 276.0571 & 12.5649 \\
\hline MW & 10.08 .17 & 22.041 & 2.694 & 0.2763 & 3.1587 & 29.7283 & 207.9945 & 321.5826 & 53.2269 \\
\hline MW & 12.08 .17 & 18.666 & 4.551 & 1.1247 & 6.0944 & 49.9189 & 246.8832 & 180.6267 & 0 \\
\hline MW & 14.08 .17 & 19.269 & 5.154 & 3.1606 & 10.8308 & 87.7027 & 518.3101 & 513.3998 & 110.1782 \\
\hline MW & 15.08 .17 & 19.918 & 1.855 & 0.1272 & 0.742 & 5.9049 & 33.331 & 84.2202 & 0 \\
\hline MW & 17.08 .17 & 21.384 & 2.978 & 0.0619 & 0.5264 & 4.8274 & 36.7475 & 36.8723 & 26.7913 \\
\hline MW & 18.08 .17 & 20.574 & 3.080 & 0.1162 & 0.6434 & 4.26 & 21.1639 & 29.4541 & 47.5157 \\
\hline MW & 19.08 .17 & 22.836 & 2.388 & 0.059 & 0.4633 & 4.7649 & 21.8155 & 29.468 & 6.373 \\
\hline MW & 21.08 .17 & 22.127 & 1.991 & 0.2617 & 3.1625 & 17.5533 & 33.4467 & 24.5892 & 6.2844 \\
\hline MW & 22.08 .17 & 21.126 & 3.011 & 0.2238 & 2.7402 & 39.8506 & 288.9442 & 225.7404 & 24.2119 \\
\hline MW & 20.07 .17 & 15.915 & 4.620 & 0.1951 & 1.2363 & 12.9435 & 50.1497 & 120.0182 & 51.0725 \\
\hline
\end{tabular}




\begin{tabular}{|c|c|c|c|c|c|c|c|c|c|}
\hline MW & 21.07 .17 & 14.153 & 3.830 & 0.0234 & 0.1781 & 1.9931 & 7.0882 & 8.4802 & 2.4772 \\
\hline MW & 22.07 .17 & 15.603 & 2.559 & 0.0059 & 0.0165 & 0.0728 & 0.2085 & 0.3042 & 0 \\
\hline MW & 23.07 .17 & 17.732 & 4.427 & 0.0219 & 0.1316 & 1.4648 & 7.4614 & 6.4594 & 12.1317 \\
\hline MW & 26.07 .17 & 20.989 & 2.550 & 0.1499 & 1.7981 & 9.1141 & 11.8906 & 1.866 & 0 \\
\hline MW & 27.07 .17 & 21.563 & 2.092 & 0.1436 & 2.5401 & 16.6638 & 30.1571 & 9.0439 & 0 \\
\hline MW & 28.07 .17 & 22.325 & 2.318 & 0.1674 & 2.6065 & 12.0603 & 35.6525 & 24.0122 & 0 \\
\hline MW & 29.07 .17 & 21.971 & 4.438 & 0.2055 & 2.4908 & 42.7172 & 163.4428 & 109.8901 & 0 \\
\hline MW & 30.07 .17 & 19.823 & 4.874 & 0.0576 & 0.4659 & 2.2444 & 5.989 & 7.0296 & 0 \\
\hline BS & 03.08 .17 & 16.832 & 2.182 & 0.0137 & 0.077 & 0.3143 & 0.7407 & 3.8354 & 0 \\
\hline BS & 04.08 .17 & 18.734 & 3.251 & 0.0193 & 0.1162 & 0.5597 & 1.6363 & 1.2249 & 0 \\
\hline $\mathrm{BS}$ & 05.08 .17 & 21.383 & 5.379 & 0.0506 & 0.6417 & 3.5234 & 8.293 & 9.3819 & 3.3068 \\
\hline $\mathrm{BS}$ & 06.08 .17 & 22.347 & 6.790 & 0.3858 & 5.0476 & 45.0073 & 159.0974 & 75.0699 & 26.4677 \\
\hline BS & 07.08 .17 & 22.706 & 4.842 & 0.6379 & 9.1193 & 51.2209 & 130.3214 & 80.8477 & 0 \\
\hline $\mathrm{BS}$ & 08.08 .17 & 22.889 & 4.303 & 0.7554 & 6.9688 & 36.7909 & 125.2447 & 88.1866 & 0 \\
\hline BS & 09.08 .17 & 22.064 & 3.045 & 0.3203 & 3.0439 & 13.5882 & 33.253 & 16.5779 & 21.672 \\
\hline BS & 10.08 .17 & 22.041 & 2.694 & 0.194 & 2.0181 & 8.6547 & 17.7228 & 10.3509 & 0 \\
\hline BS & 12.08 .17 & 18.666 & 4.551 & 0.037 & 0.3435 & 2.2437 & 8.2023 & 6.6773 & 4.2183 \\
\hline $\mathrm{BS}$ & 14.08 .17 & 19.269 & 5.154 & 0.1175 & 0.9683 & 7.6959 & 32.4113 & 37.7385 & 3.5343 \\
\hline $\mathrm{BS}$ & 16.08 .17 & 22.284 & 1.523 & 0.0036 & 0.0253 & 0.1168 & 0 & 0 & 0 \\
\hline $\mathrm{BS}$ & 18.08 .17 & 20.574 & 3.080 & 0.0403 & 0.2139 & 1.0044 & 3.4598 & 2.1027 & 0 \\
\hline $\mathrm{BS}$ & 20.08 .17 & 23.049 & 2.321 & 0.2178 & 2.2909 & 13.381 & 48.7953 & 48.1988 & 0 \\
\hline BS & 21.08 .17 & 23.049 & 1.991 & 0.3135 & 4.0807 & 21.3125 & 59.5899 & 52.2078 & 121.0427 \\
\hline BS & 24.07 .17 & 20.758 & 3.883 & 0.2912 & 3.0925 & 14.4286 & 33.4588 & 28.8779 & 0 \\
\hline BS & 25.07 .17 & 20.784 & 2.055 & 0.3448 & 2.9782 & 23.8669 & 15.4882 & 2.9016 & 0 \\
\hline $\mathrm{BS}$ & 26.07 .17 & 20.989 & 2.550 & 0.1655 & 1.8011 & 7.0431 & 15.266 & 3.2846 & 0 \\
\hline $\mathrm{BS}$ & 27.07 .17 & 21.563 & 2.092 & 0.2367 & 2.3747 & 9.1715 & 14.3749 & 2.402 & 0 \\
\hline $\mathrm{BS}$ & 28.07 .17 & 22.325 & 2.318 & 0.2228 & 2.2278 & 8.9356 & 9.8436 & 1.0245 & 0 \\
\hline $\mathrm{BS}$ & 29.07 .17 & 21.971 & 4.438 & 0.1944 & 2.3734 & 11.0921 & 20.7425 & 10.5312 & 4.1926 \\
\hline $\mathrm{BS}$ & 30.07 .17 & 19.823 & 4.874 & 0.0469 & 0.5442 & 2.8858 & 9.4036 & 5.3719 & 0 \\
\hline
\end{tabular}


Table S 4: A daily basis number deposition rate $\left(\mathbf{1} /\left(\mathbf{m}^{2} \mathbf{d}\right)\right)$ shown in each interval measured by

Horizontal deposition samplers.

\author{
$\mathrm{MW}=\mathrm{MWAC}, \mathrm{BS}=\mathrm{BSNE}$
}

\begin{tabular}{|c|c|c|c|c|c|c|c|c|c|}
\hline \multirow{3}{*}{$\begin{array}{l}\text { Samp. } \\
\text { Id. }\end{array}$} & \multirow{3}{*}{ Date } & \multirow{3}{*}{$\begin{array}{l}\text { Temp. } \\
\left({ }^{\circ} \mathrm{C}\right)\end{array}$} & \multirow{3}{*}{$\begin{array}{c}\text { Wind } \\
\text { Speed } \\
(\mathrm{m} / \mathrm{s})\end{array}$} & \multicolumn{6}{|c|}{ Number deposition rate $1 /\left(\mathrm{m}^{2} \mathrm{~d}\right)$} \\
\hline & & & & \multicolumn{6}{|c|}{ Size interval (aerodynamic diameter) } \\
\hline & & & & $1-2 \mu \mathrm{m}$ & $2-4 \mu \mathrm{m}$ & $4-8 \mu \mathrm{m}$ & $8-16 \mu \mathrm{m}$ & $\begin{array}{c}16-32 \\
\mu \mathrm{m}\end{array}$ & $32-64 \mu \mathrm{m}$ \\
\hline MW & 02.08 .17 & 15.997 & 2.055 & $1.10 \mathrm{E}+07$ & $9.09 \mathrm{E}+06$ & $1.02 \mathrm{E}+07$ & $7.47 \mathrm{E}+06$ & $7.70 \mathrm{E}+05$ & 0 \\
\hline MW & 04.08 .17 & 18.734 & 3.251 & $7.12 \mathrm{E}+06$ & $4.26 \mathrm{E}+06$ & $2.35 \mathrm{E}+06$ & $4.20 \mathrm{E}+05$ & $8.40 \mathrm{E}+04$ & 0 \\
\hline MW & 08.08 .17 & 22.889 & 4.303 & $1.06 \mathrm{E}+08$ & $1.29 \mathrm{E}+08$ & $1.71 \mathrm{E}+08$ & $1.86 \mathrm{E}+08$ & $3.41 \mathrm{E}+07$ & $2.00 \mathrm{E}+05$ \\
\hline MW & 09.08 .17 & 22.064 & 3.045 & $7.20 \mathrm{E}+07$ & $9.20 \mathrm{E}+07$ & $1.40 \mathrm{E}+08$ & $1.49 \mathrm{E}+08$ & $1.66 \mathrm{E}+07$ & $1.00 \mathrm{E}+05$ \\
\hline MW & 10.08 .17 & 22.041 & 2.694 & $4.75 \mathrm{E}+07$ & $8.06 \mathrm{E}+07$ & $8.56 \mathrm{E}+07$ & $8.75 \mathrm{E}+07$ & $2.04 \mathrm{E}+07$ & $4.80 \mathrm{E}+05$ \\
\hline MW & 12.08 .17 & 18.666 & 4.551 & $2.21 \mathrm{E}+08$ & $1.76 \mathrm{E}+08$ & $1.47 \mathrm{E}+08$ & $1.07 \mathrm{E}+08$ & $1.42 \mathrm{E}+07$ & 0 \\
\hline MW & 14.08 .17 & 19.269 & 5.154 & $6.09 \mathrm{E}+08$ & $3.49 \mathrm{E}+08$ & $2.46 \mathrm{E}+08$ & $2.21 \mathrm{E}+08$ & $3.26 \mathrm{E}+07$ & $1.20 \mathrm{E}+06$ \\
\hline MW & 15.08 .17 & 19.918 & 1.855 & $2.57 \mathrm{E}+07$ & $2.04 \mathrm{E}+07$ & $1.67 \mathrm{E}+07$ & $1.33 \mathrm{E}+07$ & $4.02 \mathrm{E}+06$ & 0 \\
\hline MW & 17.08 .17 & 21.384 & 2.978 & $1.10 \mathrm{E}+07$ & $1.33 \mathrm{E}+07$ & $1.41 \mathrm{E}+07$ & $1.58 \mathrm{E}+07$ & $2.08 \mathrm{E}+06$ & $9.00 \mathrm{E}+04$ \\
\hline MW & 18.08 .17 & 20.574 & 3.080 & $2.22 \mathrm{E}+07$ & $1.83 \mathrm{E}+07$ & $1.27 \mathrm{E}+07$ & $9.05 \mathrm{E}+06$ & $1.76 \mathrm{E}+06$ & $2.30 \mathrm{E}+05$ \\
\hline MW & 19.08 .17 & 22.836 & 2.388 & $1.08 \mathrm{E}+07$ & $1.12 \mathrm{E}+07$ & $1.36 \mathrm{E}+07$ & $1.00 \mathrm{E}+07$ & $1.82 \mathrm{E}+06$ & $8.00 \mathrm{E}+04$ \\
\hline MW & 21.08 .17 & 22.127 & 1.991 & $4.54 \mathrm{E}+07$ & $7.94 \mathrm{E}+07$ & $6.27 \mathrm{E}+07$ & $1.72 \mathrm{E}+07$ & $1.32 \mathrm{E}+06$ & $7.00 \mathrm{E}+04$ \\
\hline MW & 22.08 .17 & 21.126 & 3.011 & $4.07 \mathrm{E}+07$ & $6.93 E+07$ & $1.07 \mathrm{E}+08$ & $1.24 \mathrm{E}+08$ & $1.49 \mathrm{E}+07$ & $1.00 \mathrm{E}+05$ \\
\hline MW & 20.07 .17 & 15.915 & 4.620 & $3.67 \mathrm{E}+07$ & $3.23 \mathrm{E}+07$ & $3.71 \mathrm{E}+07$ & $2.25 \mathrm{E}+07$ & $5.07 \mathrm{E}+06$ & $2.90 \mathrm{E}+05$ \\
\hline MW & 21.07 .17 & 14.153 & 3.830 & $2.73 \mathrm{E}+07$ & $2.67 \mathrm{E}+07$ & $3.21 \mathrm{E}+07$ & $1.68 \mathrm{E}+07$ & $2.74 \mathrm{E}+06$ & $1.50 \mathrm{E}+05$ \\
\hline MW & 22.07 .17 & 15.603 & 2.559 & $1.33 \mathrm{E}+06$ & $4.42 \mathrm{E}+05$ & $1.63 \mathrm{E}+05$ & $9.30 \mathrm{E}+04$ & $2.30 \mathrm{E}+04$ & 0 \\
\hline MW & 23.07 .17 & 17.732 & 4.427 & $4.31 \mathrm{E}+06$ & $3.82 \mathrm{E}+06$ & $3.87 \mathrm{E}+06$ & $3.55 \mathrm{E}+06$ & $4.33 \mathrm{E}+05$ & $2.70 \mathrm{E}+04$ \\
\hline MW & 26.07 .17 & 20.989 & 2.550 & $2.66 \mathrm{E}+07$ & $4.53 \mathrm{E}+07$ & $3.34 \mathrm{E}+07$ & $6.39 \mathrm{E}+06$ & $1.80 \mathrm{E}+05$ & 0 \\
\hline MW & 27.07 .17 & 21.563 & 2.092 & $2.50 \mathrm{E}+07$ & $6.16 \mathrm{E}+07$ & $5.73 \mathrm{E}+07$ & $1.62 \mathrm{E}+07$ & $5.60 \mathrm{E}+05$ & 0 \\
\hline MW & 28.07 .17 & 22.325 & 2.318 & $2.81 \mathrm{E}+07$ & $6.41 \mathrm{E}+07$ & $4.25 \mathrm{E}+07$ & $1.51 \mathrm{E}+07$ & $1.82 \mathrm{E}+06$ & 0 \\
\hline MW & 29.07 .17 & 21.971 & 4.438 & $3.80 \mathrm{E}+07$ & $5.98 \mathrm{E}+07$ & $1.13 \mathrm{E}+08$ & $8.17 \mathrm{E}+07$ & $7.80 \mathrm{E}+06$ & 0 \\
\hline MW & 30.07 .17 & 19.823 & 4.874 & $1.20 \mathrm{E}+07$ & $1.23 \mathrm{E}+07$ & $8.23 E+06$ & $2.59 \mathrm{E}+06$ & $3.50 \mathrm{E}+05$ & 0 \\
\hline $\mathrm{BS}$ & 03.08 .17 & 16.832 & 2.182 & $2.67 \mathrm{E}+06$ & $2.17 \mathrm{E}+06$ & $1.09 \mathrm{E}+06$ & $3.29 \mathrm{E}+05$ & $9.90 \mathrm{E}+04$ & 0 \\
\hline BS & 04.08 .17 & 18.734 & 3.251 & $4.43 \mathrm{E}+06$ & $3.20 \mathrm{E}+06$ & $1.92 \mathrm{E}+06$ & $6.94 \mathrm{E}+05$ & $9.90 \mathrm{E}+04$ & 0 \\
\hline BS & 05.08 .17 & 21.383 & 5.379 & $9.16 \mathrm{E}+06$ & $1.64 \mathrm{E}+07$ & $1.20 \mathrm{E}+07$ & $4.15 E+06$ & $4.90 \mathrm{E}+05$ & $4.00 \mathrm{E}+04$ \\
\hline $\mathrm{BS}$ & 06.08 .17 & 22.347 & 6.790 & $6.61 \mathrm{E}+06$ & $1.27 \mathrm{E}+07$ & $1.39 \mathrm{E}+07$ & $7.15 \mathrm{E}+06$ & $6.30 \mathrm{E}+05$ & $3.00 \mathrm{E}+04$ \\
\hline $\mathrm{BS}$ & 07.08 .17 & 22.706 & 4.842 & $1.06 \mathrm{E}+08$ & $2.32 \mathrm{E}+08$ & $1.72 \mathrm{E}+08$ & $5.82 \mathrm{E}+07$ & $6.40 \mathrm{E}+06$ & 0 \\
\hline BS & 08.08 .17 & 22.889 & 4.303 & $1.44 \mathrm{E}+08$ & $1.79 \mathrm{E}+08$ & $1.29 \mathrm{E}+08$ & $5.15 \mathrm{E}+07$ & $7.70 \mathrm{E}+06$ & 0 \\
\hline BS & 09.08 .17 & 22.064 & 3.045 & $5.65 \mathrm{E}+07$ & $7.88 \mathrm{E}+07$ & $5.07 \mathrm{E}+07$ & $1.62 \mathrm{E}+07$ & $1.01 \mathrm{E}+06$ & $2.00 \mathrm{E}+05$ \\
\hline BS & 10.08 .17 & 22.041 & 2.694 & $3.35 \mathrm{E}+07$ & $5.33 \mathrm{E}+07$ & $3.11 \mathrm{E}+07$ & $9.35 \mathrm{E}+06$ & $8.30 \mathrm{E}+05$ & 0 \\
\hline BS & 12.08 .17 & 18.666 & 4.551 & $7.11 \mathrm{E}+06$ & $8.52 \mathrm{E}+06$ & $6.52 \mathrm{E}+06$ & $3.50 \mathrm{E}+06$ & $5.08 \mathrm{E}+05$ & $2.80 \mathrm{E}+04$ \\
\hline BS & 14.08 .17 & 19.269 & 5.154 & $2.45 \mathrm{E}+07$ & $2.59 \mathrm{E}+07$ & $2.36 \mathrm{E}+07$ & $1.39 \mathrm{E}+07$ & $2.10 \mathrm{E}+06$ & $4.00 \mathrm{E}+04$ \\
\hline $\mathrm{BS}$ & 16.08 .17 & 22.284 & 1.523 & $6.98 \mathrm{E}+05$ & $7.62 \mathrm{E}+05$ & $4.44 \mathrm{E}+05$ & 0 & 0 & 0 \\
\hline BS & 18.08 .17 & 20.574 & 3.080 & $8.63 \mathrm{E}+06$ & $6.30 \mathrm{E}+06$ & $3.38 \mathrm{E}+06$ & $1.63 \mathrm{E}+06$ & $2.08 \mathrm{E}+05$ & 0 \\
\hline
\end{tabular}




\begin{tabular}{|c|c|c|c|c|c|c|c|c|c|} 
BS & 20.08 .17 & 23.049 & 2.321 & $3.65 \mathrm{E}+07$ & $6.08 \mathrm{E}+07$ & $4.45 \mathrm{E}+07$ & $2.19 \mathrm{E}+07$ & $3.23 \mathrm{E}+06$ & 0 \\
\hline BS & 21.08 .17 & 23.049 & 1.991 & $5.14 \mathrm{E}+07$ & $1.01 \mathrm{E}+08$ & $7.45 \mathrm{E}+07$ & $2.65 \mathrm{E}+07$ & $3.30 \mathrm{E}+06$ & $2.00 \mathrm{E}+05$ \\
\hline BS & 24.07 .17 & 20.758 & 3.883 & $5.28 \mathrm{E}+07$ & $7.98 \mathrm{E}+07$ & $4.97 \mathrm{E}+07$ & $1.60 \mathrm{E}+07$ & $1.96 \mathrm{E}+06$ & 0 \\
\hline BS & 25.07 .17 & 20.784 & 2.055 & $6.03 \mathrm{E}+07$ & $7.79 \mathrm{E}+07$ & $3.61 \mathrm{E}+07$ & $8.75 \mathrm{E}+06$ & $2.40 \mathrm{E}+05$ & 0 \\
\hline BS & 26.07 .17 & 20.989 & 2.550 & $2.95 \mathrm{E}+07$ & $4.74 \mathrm{E}+07$ & $2.66 \mathrm{E}+07$ & $7.58 \mathrm{E}+06$ & $2.90 \mathrm{E}+05$ & 0 \\
\hline BS & 27.07 .17 & 21.563 & 2.092 & $4.11 \mathrm{E}+07$ & $6.26 \mathrm{E}+07$ & $3.46 \mathrm{E}+07$ & $7.21 \mathrm{E}+06$ & $2.00 \mathrm{E}+05$ & 0 \\
\hline BS & 28.07 .17 & 22.325 & 2.318 & $3.95 \mathrm{E}+07$ & $5.91 \mathrm{E}+07$ & $3.33 \mathrm{E}+07$ & $5.38 \mathrm{E}+06$ & $1.00 \mathrm{E}+05$ & 0 \\
\hline BS & 29.07 .17 & 21.971 & 4.438 & $3.27 \mathrm{E}+07$ & $6.05 \mathrm{E}+07$ & $3.87 \mathrm{E}+07$ & $1.03 \mathrm{E}+07$ & $7.50 \mathrm{E}+05$ & $5.00 \mathrm{E}+04$ \\
\hline BS & 30.07 .17 & 19.823 & 4.874 & $8.72 \mathrm{E}+06$ & $1.35 \mathrm{E}+07$ & $1.00 \mathrm{E}+07$ & $4.23 \mathrm{E}+06$ & $4.70 \mathrm{E}+05$ & 0 \\
\hline
\end{tabular}

Table S 5: A daily basis upward and downward mass deposition rate $\left(\mathbf{m g} /\left(\mathbf{m}^{2} \mathbf{d}\right)\right)$ shown in each interval measured by Flat plate sampler $(25 \mathrm{~mm})$ specified in size intervals $(\mu \mathrm{m})$.

FP_U=Upward deposition rate, FP_D=Downward deposition rate

\begin{tabular}{|c|c|c|c|c|c|c|c|c|c|}
\hline & \multirow{2}{*}{$\begin{array}{c}\text { Samp. } \\
\text { Id. }\end{array}$} & \multirow{2}{*}{$\begin{array}{l}\text { Demp. } \\
\left({ }^{\circ} \mathrm{C}\right)\end{array}$} & \multirow{2}{*}{$\begin{array}{l}\text { Wind } \\
\text { speed } \\
(\mathrm{m} / \mathrm{s})\end{array}$} & & \multicolumn{6}{|c|}{ Mass deposition rate $\left(\mathrm{mg} /\left(\mathrm{m}^{2} \mathrm{~d}\right)\right)$} \\
\cline { 6 - 11 } & & & & $1-2 \mu \mathrm{m}$ & $2-4 \mu \mathrm{m}$ & $4-8 \mu \mathrm{m}$ & $8-16 \mu \mathrm{m}$ & $\begin{array}{c}16-32 \\
\mu \mathrm{m}\end{array}$ & $\begin{array}{c}32-64 \\
\mu \mathrm{m}\end{array}$ \\
\hline FP_U & 20.07 .17 & 15.915 & 4.620 & 0.006 & 0.0126 & 0.0071 & 0.1607 & 0 & 0 \\
\hline FP_U & 21.07 .17 & 14.153 & 3.830 & 0.0085 & 0.0128 & 0.1135 & 0.2452 & 0 & 0 \\
\hline FP_U & 24.07 .17 & 20.758 & 3.883 & 0.0129 & 0.0456 & 0.2431 & 0.5339 & 0 & 0 \\
\hline FP_U & 25.07 .17 & 20.784 & 2.055 & 0.0251 & 0.0397 & 0.2362 & 0 & 0 & 0 \\
\hline FP_U & 26.07 .17 & 20.989 & 2.550 & 0.0104 & 0.0803 & 0.1911 & 0.0924 & 0 & 0 \\
\hline FP_U & 27.07 .17 & 21.563 & 2.092 & 0.0065 & 0.0223 & 0.0759 & 0.2792 & 0.6606 & 0 \\
\hline FP_U & 28.07 .17 & 22.325 & 2.318 & 0.0225 & 0.0375 & 0.1186 & 0.0279 & 0 & 0 \\
\hline FP_U & 29.07 .17 & 21.971 & 4.438 & 0.021 & 0.0444 & 0.1147 & 0.2817 & 0.2653 & 0 \\
\hline & & & & & & & & & \\
\hline FP_D & 20.07 .17 & 15.915 & 4.620 & 0.0153 & 0.0578 & 0.2786 & 0.8438 & 0.2835 & 0 \\
\hline FP_D & 21.07 .17 & 14.153 & 3.830 & 0.0357 & 0.1865 & 1.0712 & 3.7204 & 2.787 & 0 \\
\hline FP_D & 24.07 .17 & 20.758 & 3.883 & 0.227 & 2.7686 & 7.7674 & 5.2542 & 7.7463 & 0 \\
\hline FP_D & 25.07 .17 & 20.784 & 2.055 & 0.2505 & 2.414 & 8.6004 & 9.5165 & 4.7043 & 0 \\
\hline FP_D & 26.07 .17 & 20.989 & 2.550 & 0.1151 & 1.4906 & 5.555 & 6.5666 & 3.226 & 0 \\
\hline FP_D & 27.07 .17 & 21.563 & 2.092 & 0.1608 & 2.1048 & 8.2862 & 10.0201 & 2.0881 & 0 \\
\hline FP_D & 28.07 .17 & 22.325 & 2.318 & 0.1585 & 1.8237 & 7.0338 & 7.517 & 1.2685 & 0 \\
\hline FP_D & 29.07 .17 & 21.971 & 4.438 & 0.154 & 2.0606 & 9.0657 & 8.783 & 2.9589 & 0 \\
\hline
\end{tabular}


Table S 6: A daily basis upward and downward number deposition rate $\left(\mathbf{1} /\left(\mathbf{m}^{\mathbf{2}} \mathbf{d}\right)\right.$ shown in each interval measured by Flat plate sampler $(25 \mathrm{~mm})$.

FP_U=Upward deposition rate, FP_D=Downward deposition rate.

\begin{tabular}{|c|c|c|c|c|c|c|c|c|c|}
\hline \multirow{3}{*}{$\begin{array}{l}\text { Samp. } \\
\text { Id. }\end{array}$} & \multirow{3}{*}{ Date } & \multirow{3}{*}{$\begin{array}{c}\text { Temp } \\
\left({ }^{\circ} \mathrm{C}\right)\end{array}$} & \multirow{3}{*}{$\begin{array}{l}\text { Wind } \\
\text { speed } \\
(\mathrm{m} / \mathrm{s})\end{array}$} & \multicolumn{6}{|c|}{ Number deposition rate, $1 /\left(\mathrm{m}^{2} \mathrm{~d}\right)$} \\
\hline & & & & \multicolumn{6}{|c|}{ Size interval (aerodynamic diameter) } \\
\hline & & & & $1-2 \mu \mathrm{m}$ & $2-4 \mu \mathrm{m}$ & $4-8 \mu \mathrm{m}$ & $8-16 \mu \mathrm{m}$ & $\begin{array}{c}16-32 \\
\mu \mathrm{m}\end{array}$ & $32-64 \mu \mathrm{m}$ \\
\hline FP_U & 20.07 .17 & 15.915 & 4.620 & $1.70 \mathrm{E}+06$ & $4.60 \mathrm{E}+05$ & $3.50 \mathrm{E}+04$ & $7.10 \mathrm{E}+04$ & 0 & 0 \\
\hline FP_U & 21.07 .17 & 14.153 & 3.830 & $1.86 \mathrm{E}+06$ & $5.32 \mathrm{E}+05$ & $3.63 \mathrm{E}+05$ & $1.21 \mathrm{E}+05$ & 0 & 0 \\
\hline FP_U & 24.07 .17 & 20.758 & 3.883 & $2.68 \mathrm{E}+06$ & $1.28 \mathrm{E}+06$ & $8.35 \mathrm{E}+05$ & $3.44 \mathrm{E}+05$ & 0 & 0 \\
\hline FP_U & 25.07 .17 & 20.784 & 2.055 & $4.99 \mathrm{E}+06$ & $1.28 \mathrm{E}+06$ & $9.14 \mathrm{E}+05$ & 0 & 0 & 0 \\
\hline FP_U & 26.07.17 & 20.989 & 2.550 & $2.33 \mathrm{E}+06$ & $2.15 \mathrm{E}+06$ & $7.46 \mathrm{E}+05$ & $6.80 \mathrm{E}+04$ & 0 & 0 \\
\hline FP_U & 27.07 .17 & 21.563 & 2.092 & $1.40 \mathrm{E}+06$ & $6.74 \mathrm{E}+05$ & $2.89 \mathrm{E}+05$ & $9.60 \mathrm{E}+04$ & $2.40 \mathrm{E}+04$ & 0 \\
\hline FP_U & 28.07 .17 & 22.325 & 2.318 & $4.84 \mathrm{E}+06$ & $1.20 \mathrm{E}+06$ & $5.19 \mathrm{E}+05$ & $2.70 \mathrm{E}+04$ & 0 & 0 \\
\hline FP_U & 29.07 .17 & 21.971 & 4.438 & $4.84 \mathrm{E}+06$ & $1.20 \mathrm{E}+06$ & $5.19 \mathrm{E}+05$ & $2.70 \mathrm{E}+04$ & 0 & 0 \\
\hline FP_D & 20.07 .17 & 15.915 & 4.620 & $3.81 \mathrm{E}+06$ & $1.82 \mathrm{E}+06$ & $9.61 \mathrm{E}+05$ & $3.64 \mathrm{E}+05$ & $3.30 \mathrm{E}+04$ & 0 \\
\hline FP_D & 21.07 .17 & 14.153 & 3.830 & $7.68 \mathrm{E}+06$ & $6.33 \mathrm{E}+06$ & $3.75 \mathrm{E}+06$ & $1.84 \mathrm{E}+06$ & $1.94 \mathrm{E}+05$ & 0 \\
\hline FP_D & 24.07 .17 & 20.758 & 3.883 & $3.90 \mathrm{E}+07$ & $7.12 \mathrm{E}+07$ & $3.37 \mathrm{E}+07$ & $3.25 \mathrm{E}+06$ & $6.20 \mathrm{E}+05$ & 0 \\
\hline FP_D & 25.07 .17 & 20.784 & 2.055 & $4.43 \mathrm{E}+07$ & $6.54 \mathrm{E}+07$ & $3.30 \mathrm{E}+07$ & $5.41 \mathrm{E}+06$ & $2.70 \mathrm{E}+05$ & 0 \\
\hline FP_D & 26.07 .17 & 20.989 & 2.550 & $2.03 \mathrm{E}+07$ & $3.77 \mathrm{E}+07$ & $2.11 \mathrm{E}+07$ & $3.41 \mathrm{E}+06$ & $2.80 \mathrm{E}+05$ & 0 \\
\hline FP_D & 27.07 .17 & 21.563 & 2.092 & $2.61 \mathrm{E}+07$ & $5.22 \mathrm{E}+07$ & $3.10 \mathrm{E}+07$ & $5.26 \mathrm{E}+06$ & $2.10 \mathrm{E}+05$ & 0 \\
\hline FP_D & 28.07 .17 & 22.325 & 2.318 & $2.75 \mathrm{E}+07$ & $4.70 \mathrm{E}+07$ & $2.61 \mathrm{E}+07$ & $4.41 \mathrm{E}+06$ & $1.30 \mathrm{E}+05$ & 0 \\
\hline FP_D & 29.07.17 & 21.971 & 4.438 & $2.59 \mathrm{E}+07$ & $5.27 \mathrm{E}+07$ & $3.50 \mathrm{E}+07$ & $4.81 \mathrm{E}+06$ & $2.40 \mathrm{E}+05$ & 0 \\
\hline
\end{tabular}

Table S 7: Summary of regression analysis for correlation between calculated dust concentration and OPC measurement

\begin{tabular}{|c|c|c|c|}
\hline \multirow{3}{*}{ Sampler } & \multicolumn{3}{|c|}{ Calculated concentration vs OPC measured concentration } \\
\cline { 2 - 4 } & $\mathrm{r}^{2}$ & $\mathrm{p}$-value & slope \\
\cline { 2 - 4 } & 0.449 & 0.0241 & 0.4084 \\
Flat plate & 0.243 & 0.214 & 0.1654 \\
MWAC & 0.968 & $4.70 \mathrm{E}-08$ & 0.8046 \\
BSNE & 0.794 & 0.00127 & 0.6851 \\
Sigma-2 & & & \\
\hline
\end{tabular}




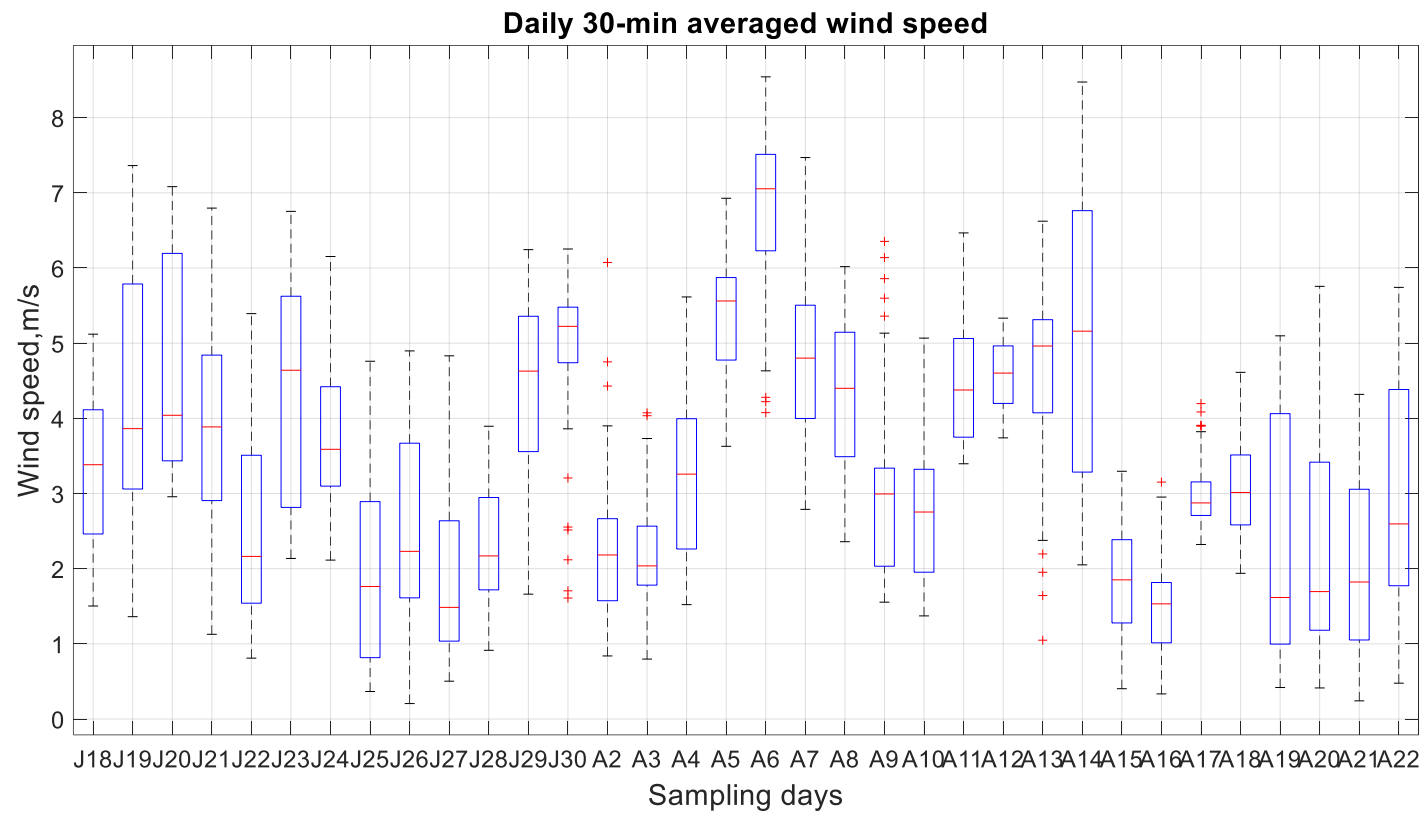

Figure S 1: Daily box-plots of 30-min averaged wind speed observed at Izaña Global Atmospheric watch Observatorio from 18/July/2017 to 23/August/2017 (e.g., each day was divided in 30-minute interval averages and then the mean and standard deviation was calculated from this data). On each blue box, the central mark is the median, the edges of the box are the 25 th and 75 th percentiles. The black vertical lines show the standard deviation ( $\mathrm{J}=\mathrm{July}, \mathrm{A}=$ August $)$.

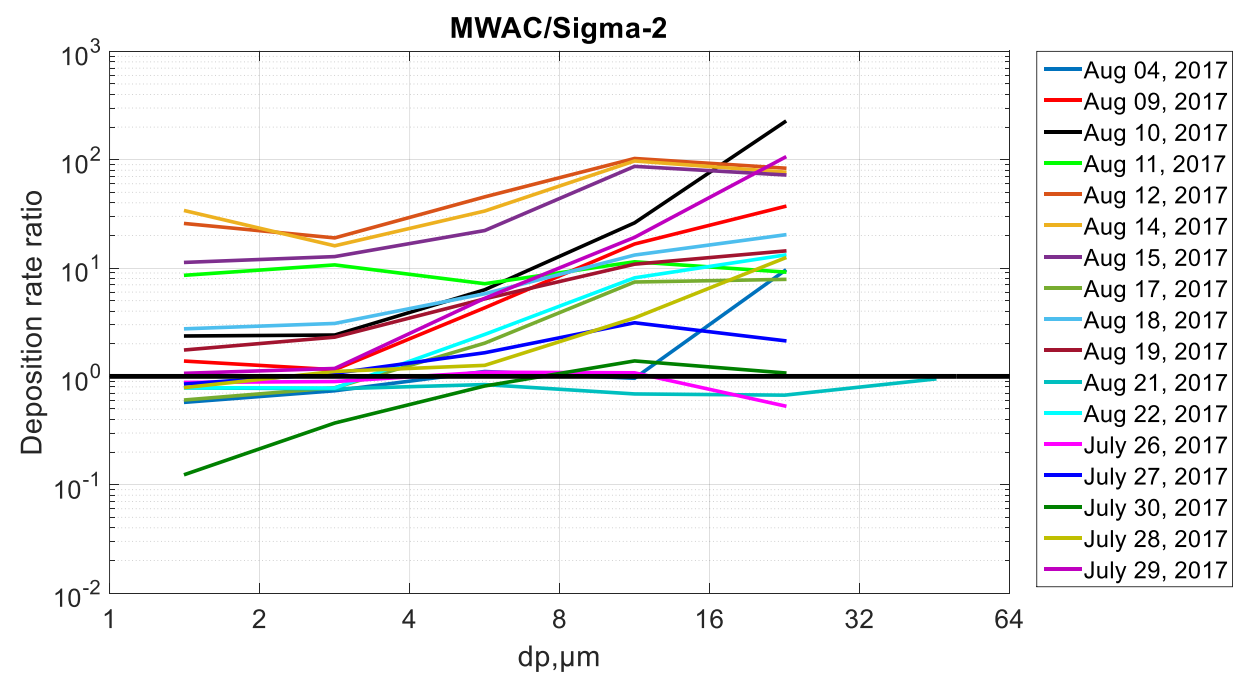


Figure S 2: Deposition rate ratio as function of particle size (MWAC/Sigma-2)

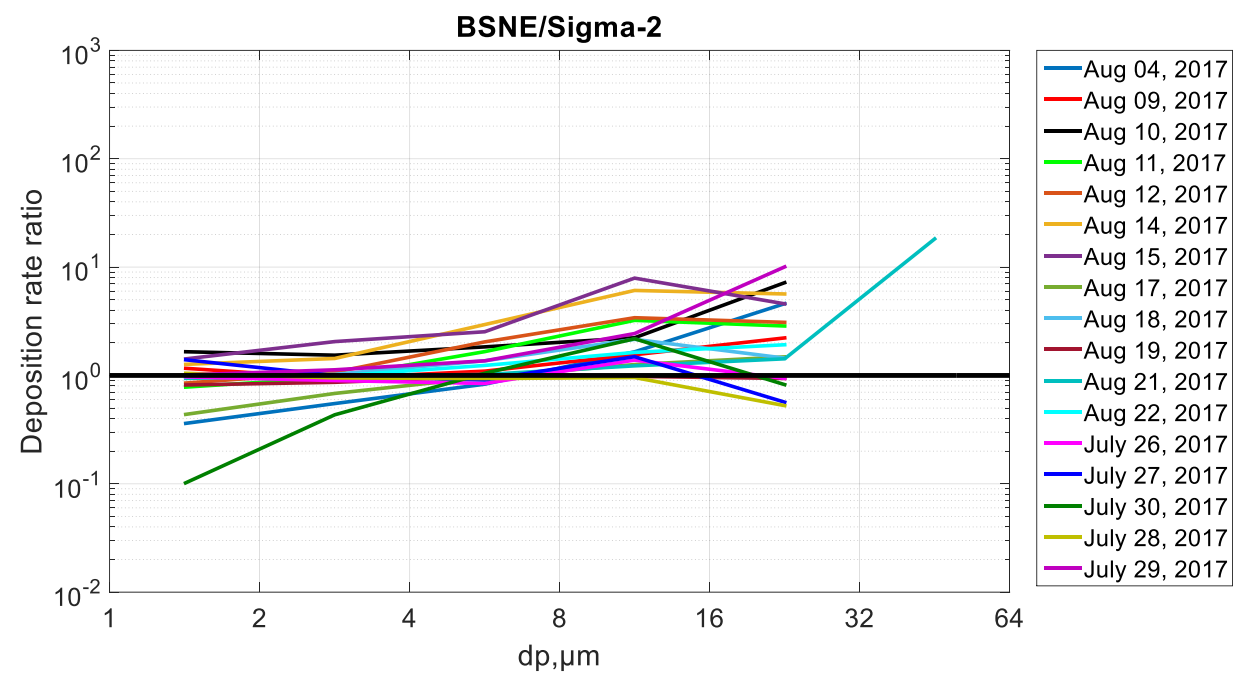

Figure S 3: Deposition rate ratio as function of particle size (BSNE/Sigma-2)

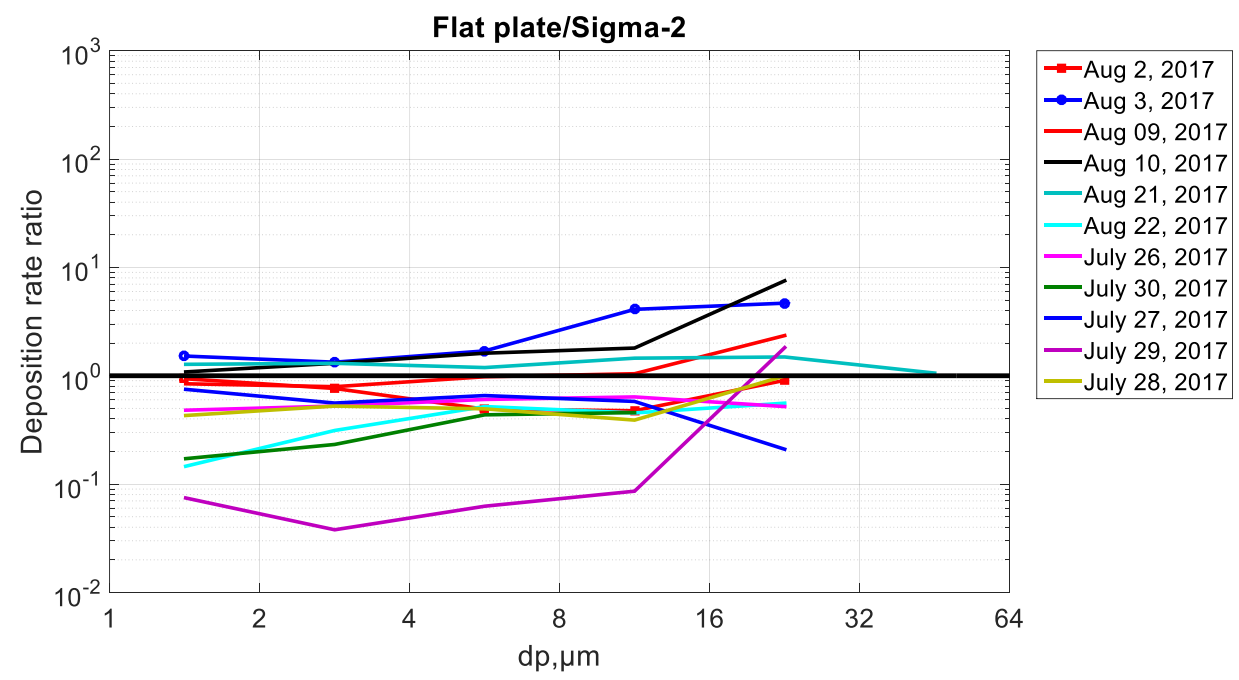


Figure S 4: Deposition rate ratio as function of particle size (Flat plate/Sigma-2)
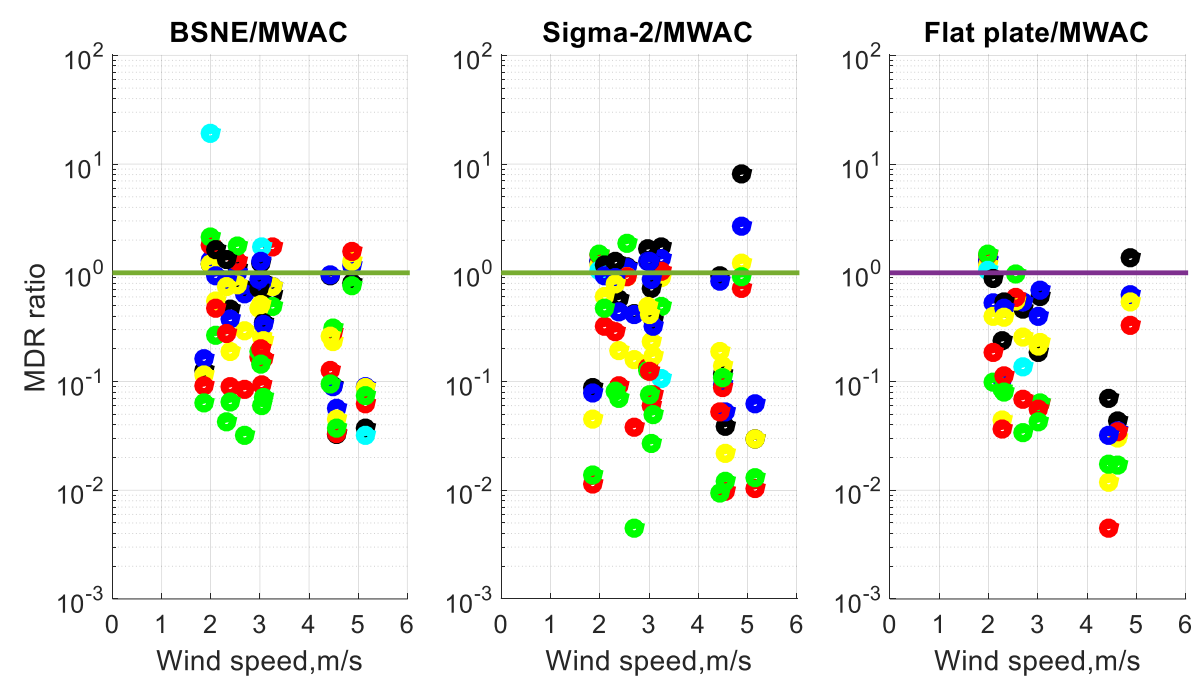

Figure S 5: Mass deposition rate (MDR) ratio as function of wind speed for different measurement days. Different colors represent deposition rates in different size intervals (black: 1-2 $\mu \mathrm{m}$; blue: 2-4 $\mu$ m; yellow: 4-8 $\mu \mathrm{m}$; red: $8-16 \mu \mathrm{m}$; green: 16-32 $\mu \mathrm{m}$; cyan: 32-64 $\mu \mathrm{m}$ ).
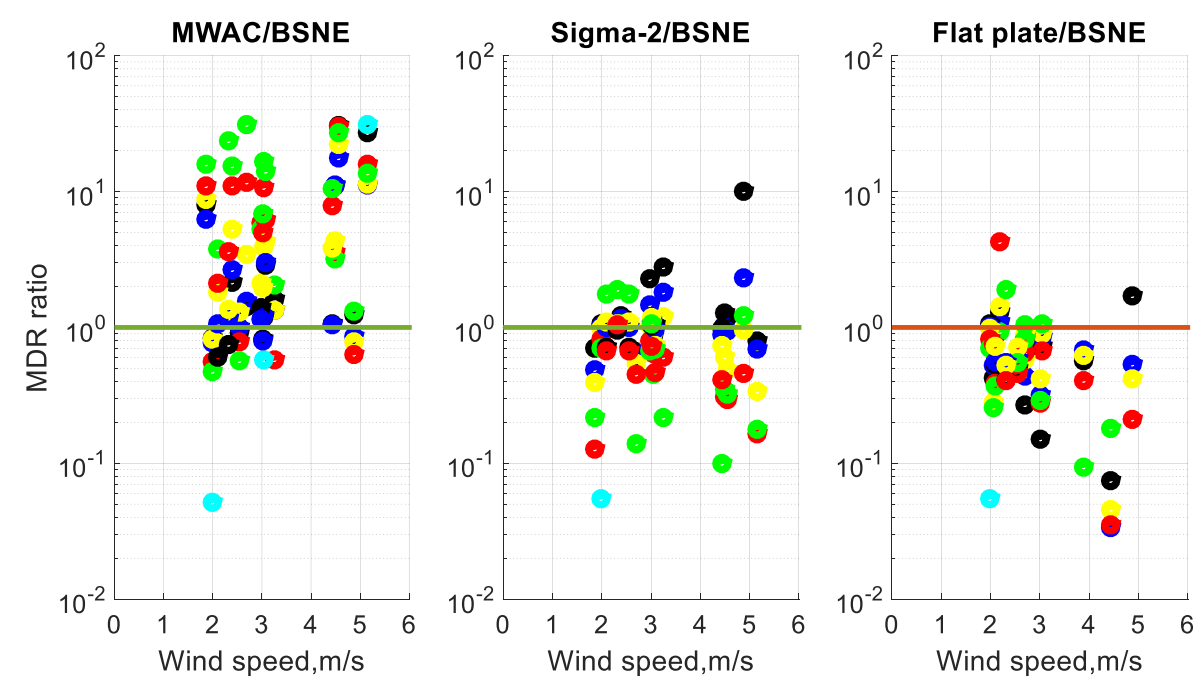

Figure S 6: Mass deposition rate (MDR) ratio as function of wind speed for different measurement days. Different colors represent deposition rates in different size intervals (black: 1-2 $\mu \mathrm{m}$; blue: $2-4 \mu \mathrm{m}$; yellow: 4-8 $\mu \mathrm{m}$; red: 8-16 $\mu \mathrm{m}$; green: 16-32 $\mu \mathrm{m}$; cyan: 32-64 $\mu \mathrm{m}$ ). 

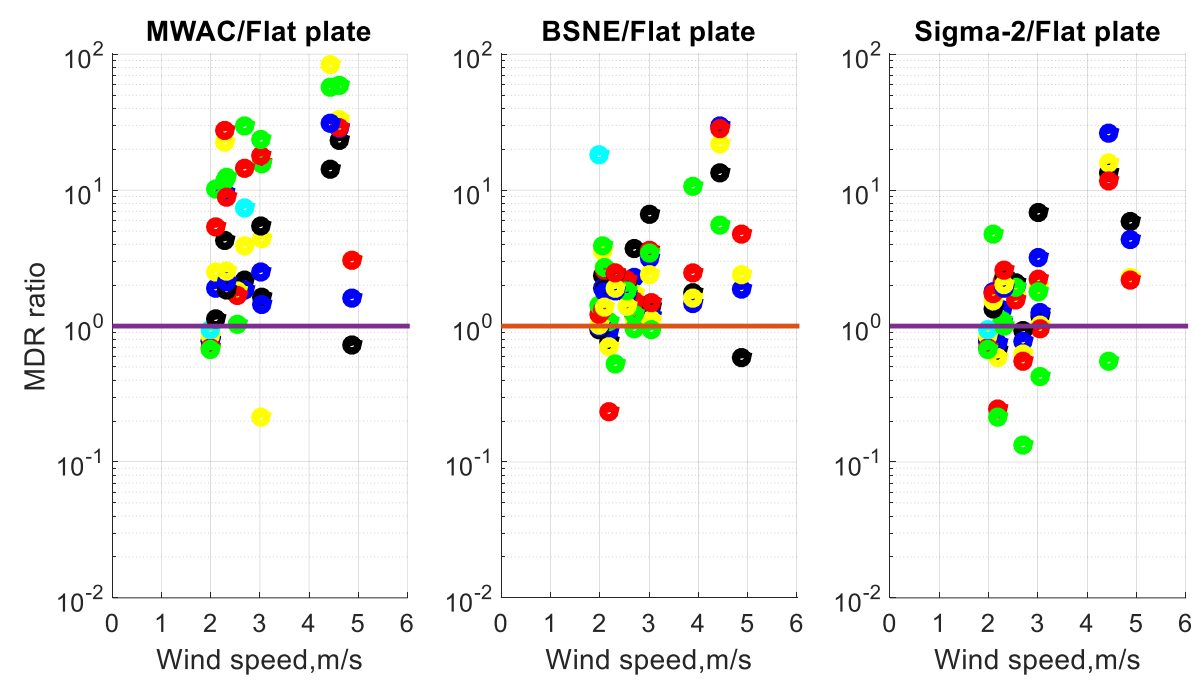

Figure S 7: Mass deposition rate (MDR) ratio as function of wind speed for different measurement days. Different colors represent deposition rates in different size intervals (black: 1-2 $\mu \mathrm{m}$; blue: 2-4 $\mu$ m; yellow: $4-8 \mu \mathrm{m}$; red: $8-16 \mu \mathrm{m}$; green: 16-32 $\mu \mathrm{m}$; cyan: 32-64 $\mu \mathrm{m}$ ).
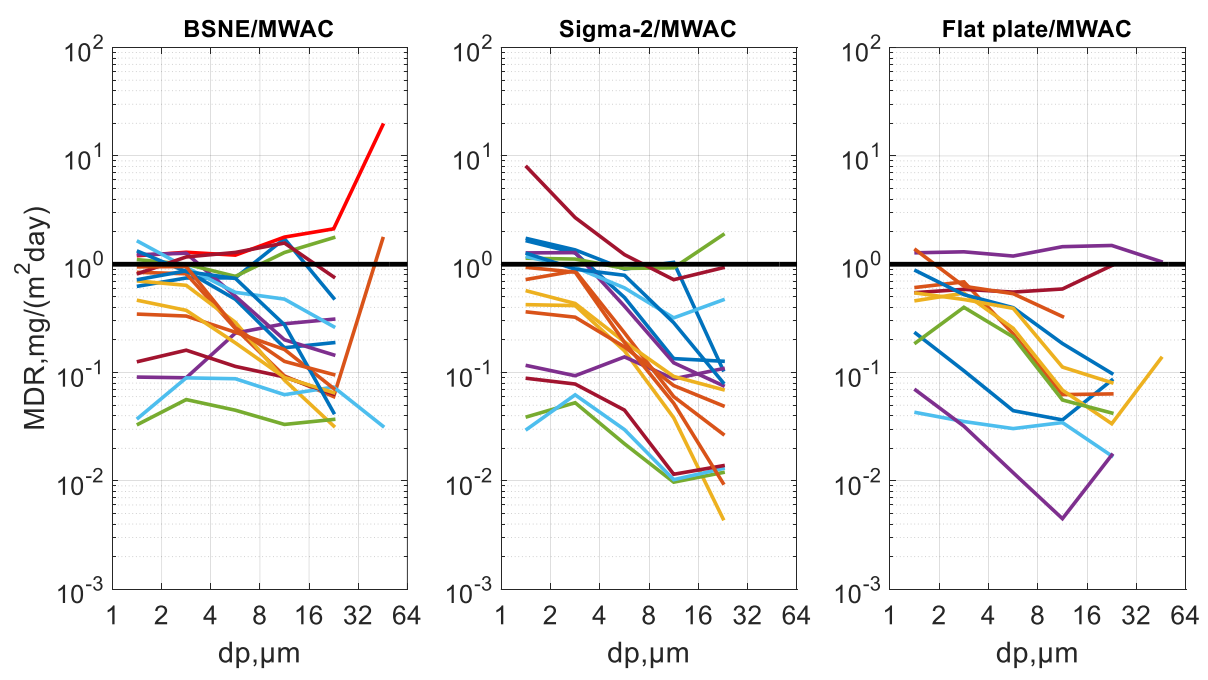

Figure S 8: Mass deposition rate (MDR) ratio as function of particle size (different colors show different measurement days). 

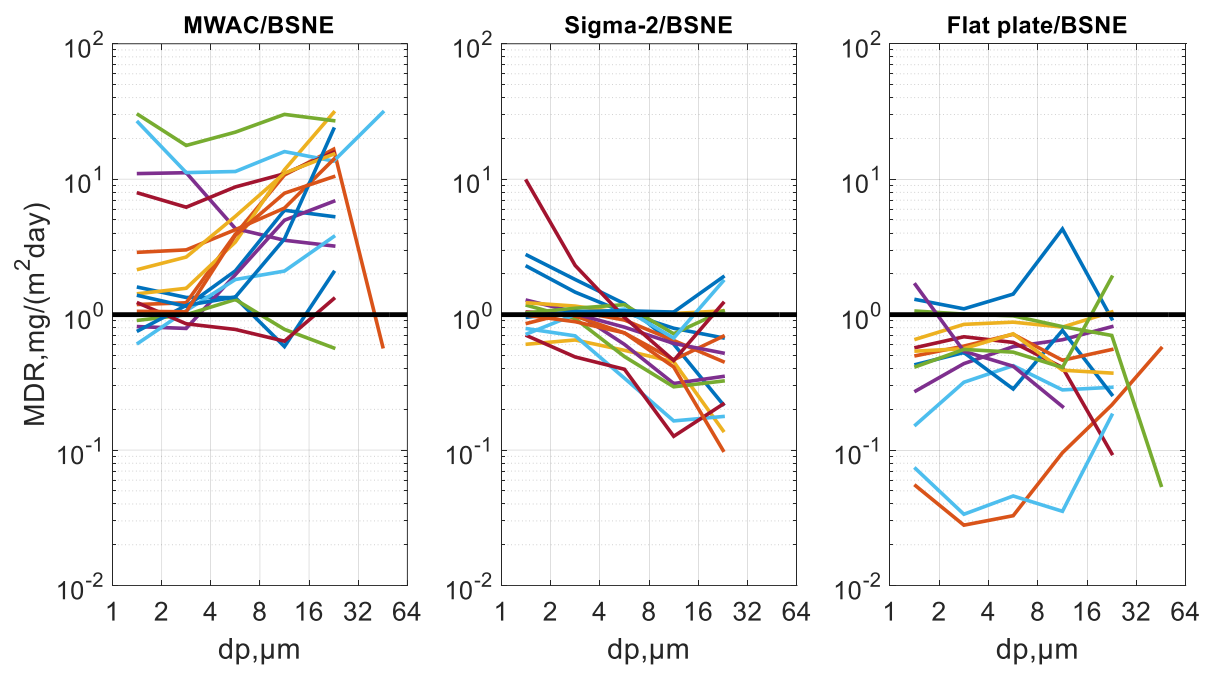

Figure S 9: Mass deposition rate (MDR) ratio as function of particle size (different colors show different measurement days).
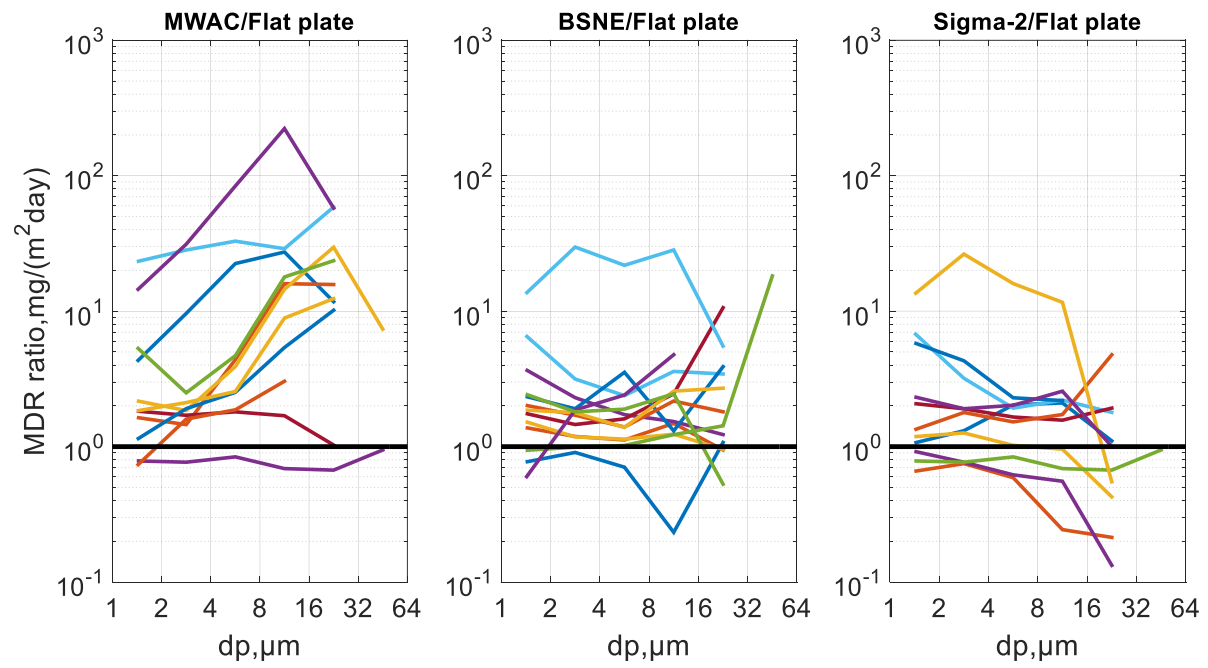

Figure S 10: Mass deposition rate (MDR) ratio as function of particle size (different colors show different measurement days). 

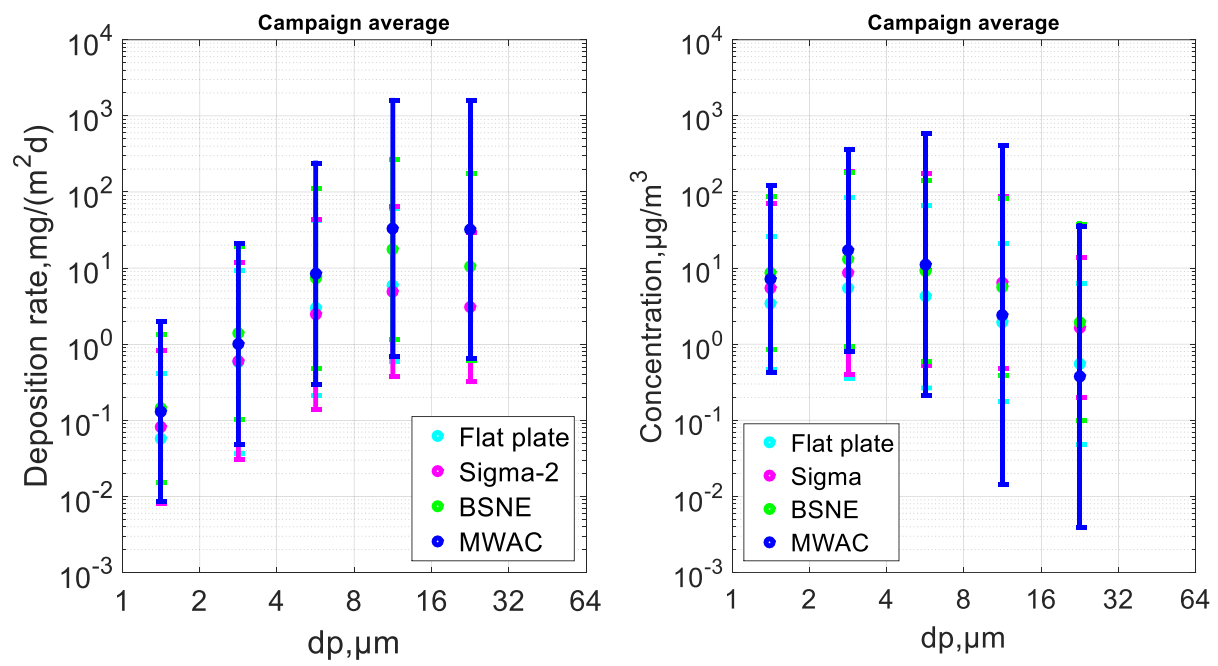

Figure S 11: Size-resolved deposition rate and mass concentration measured by different samplers (campaign data). Different deposition velocity models are used for concentration calculation (Flat plate: Piskunov; BSNE: Piskunov; MWAC: combination of Piskunov- and impaction curve). The bars show the central $95 \%$ confidence interval of the daily variation.
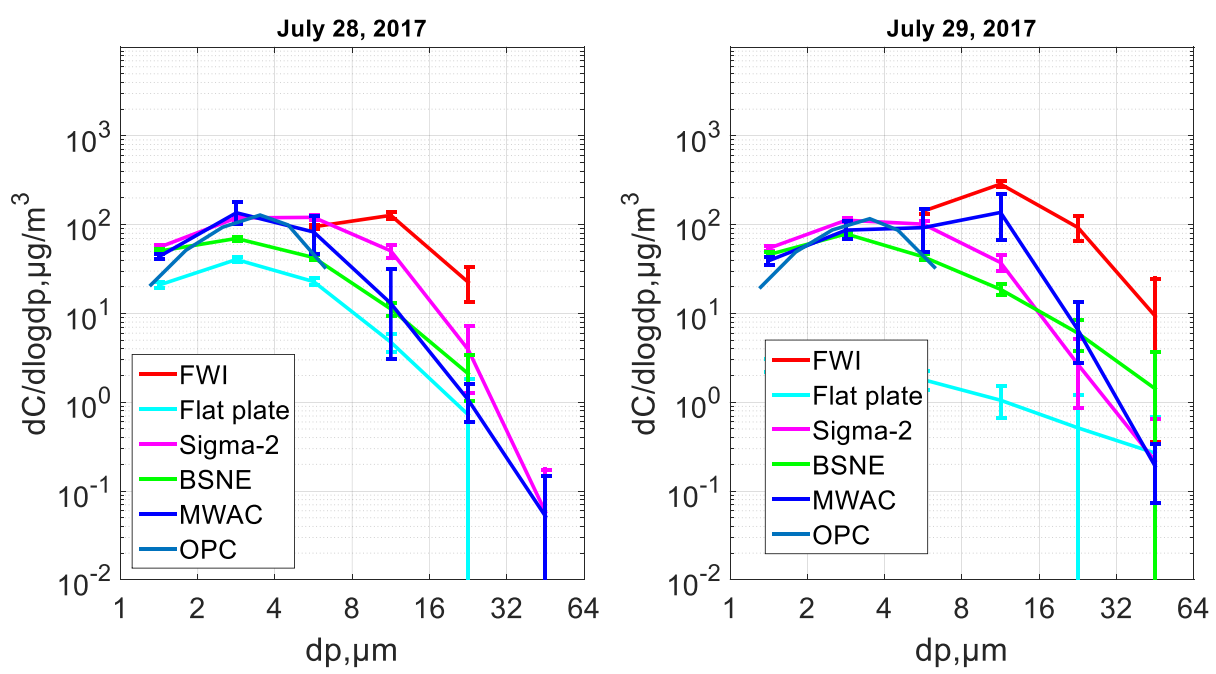

Figure S 12: Comparison of size resolved mass concentration measured by different samplers with daily average mass concentration from FWI and OPC over different measurement days. FWI measurement in each day is an average of three measurements taken every day (two days=6 samples). The bars show the central $95 \%$ confidence interval of the daily variation. 


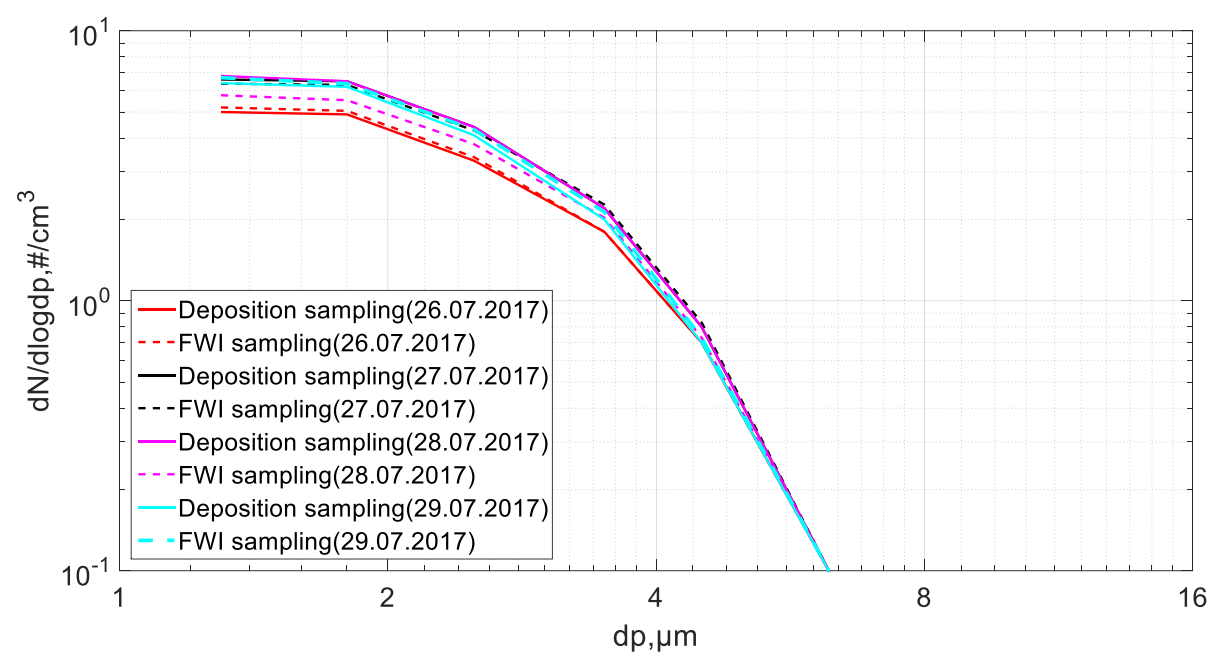

Figure S 13: An overview of the OPC measurements comparing the size distributions between the long-term (deposition) and short-term (FWI) sampling
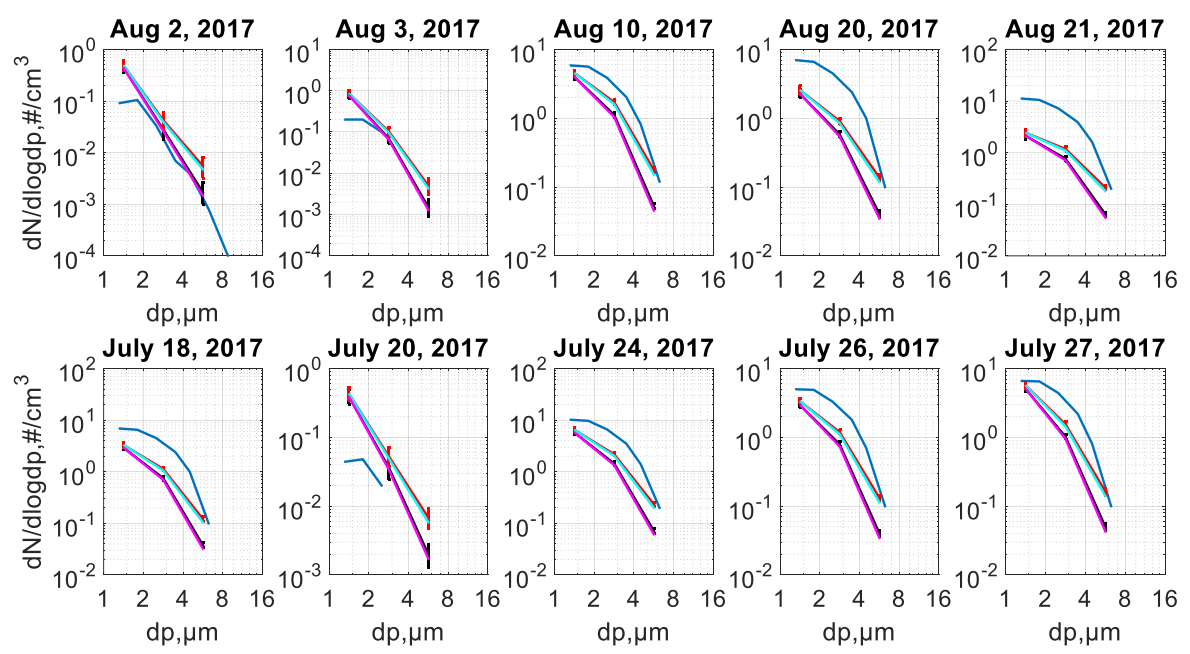

Figure S 14: Comparing number concentration calculated from deposition measurement (Flat plate sampler) (Red: Wood 1981; Black: Momentum flux; Cyan: Wood 1981-PM10 inlet and Magenta: Momentum flux-PM 10 inlet) with number concentration by OPC measurement (Blue). The bars show the central $95 \%$ confidence interval of the daily variation. 

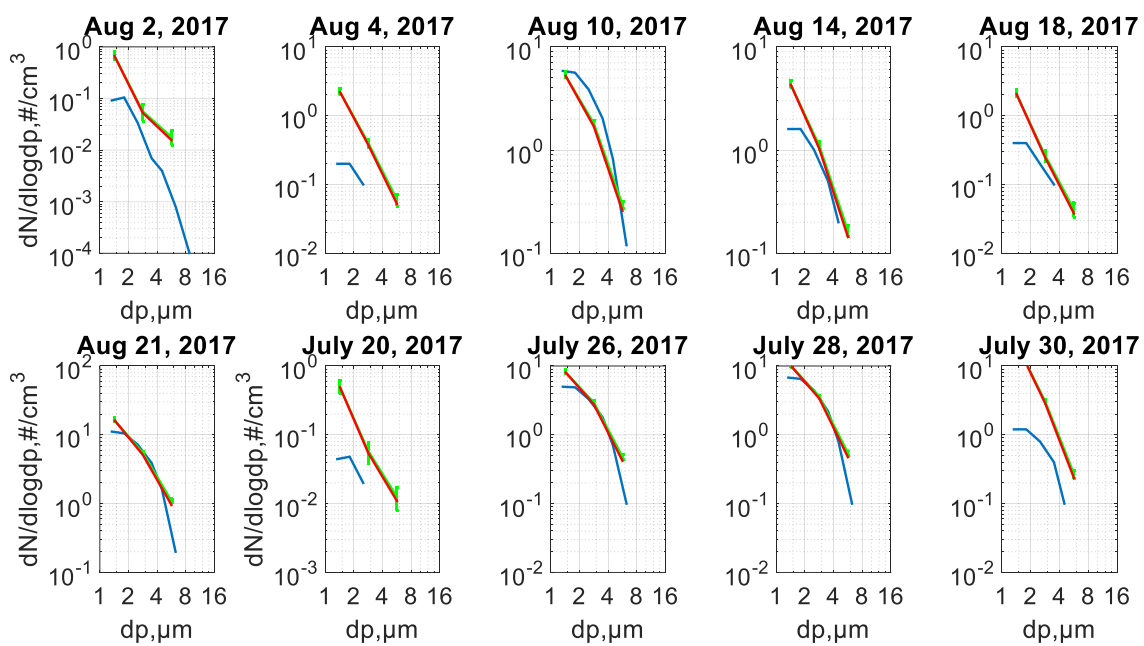

Figure S 15: Comparing number concentration calculated from deposition measurement (Sigma-2

sampler) (Green: Stokes' velocity; Red: Stokes' velocity-PM $\mathrm{PM}_{10}$ inlet using Stokes velocity. with number concentration by OPC measurement (Blue). Concentration calculation from Sigma-2 sampler considers only Stokes's velocity (without considering friction velocity). The bars show the central 95\% confidence interval of the daily variation.
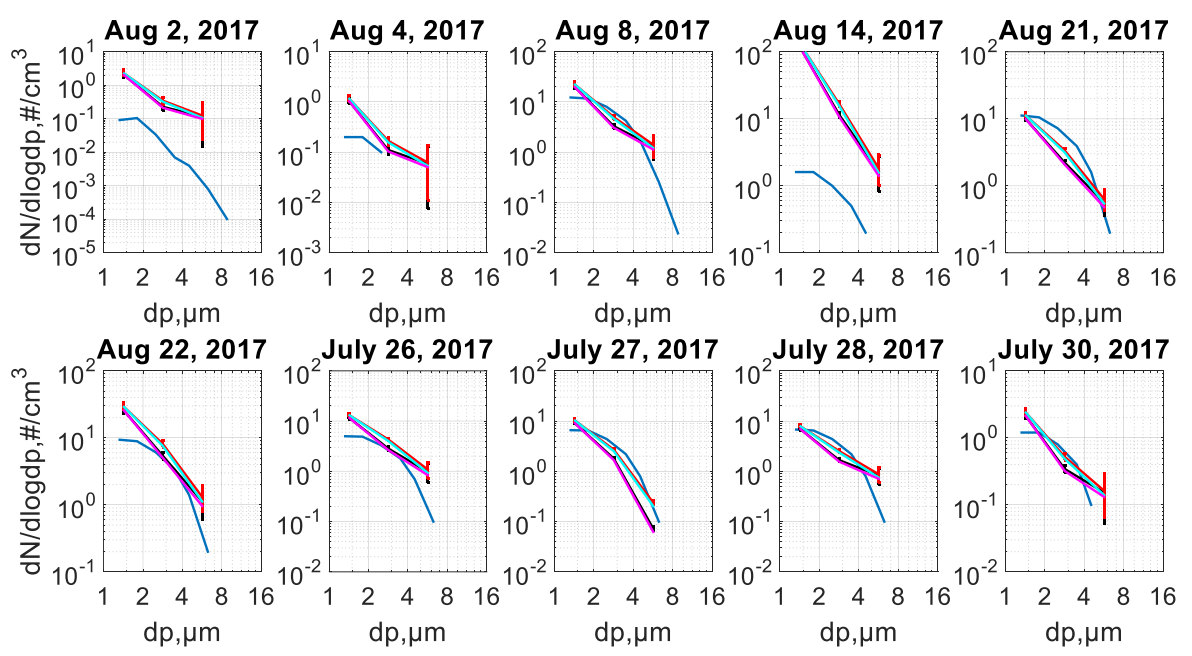

Figure S 16: Comparing number concentration calculated from deposition measurement (MWAC sampler) (Red: Wood 1981; Black: Momentum flux; Cyan: Wood 1981-PM10 inlet and Magenta: 
Momentum flux-PM $\mathrm{P}_{10}$ inlet) with number concentration by OPC measurement (Blue). The bars show the central $95 \%$ confidence interval of the daily variation.
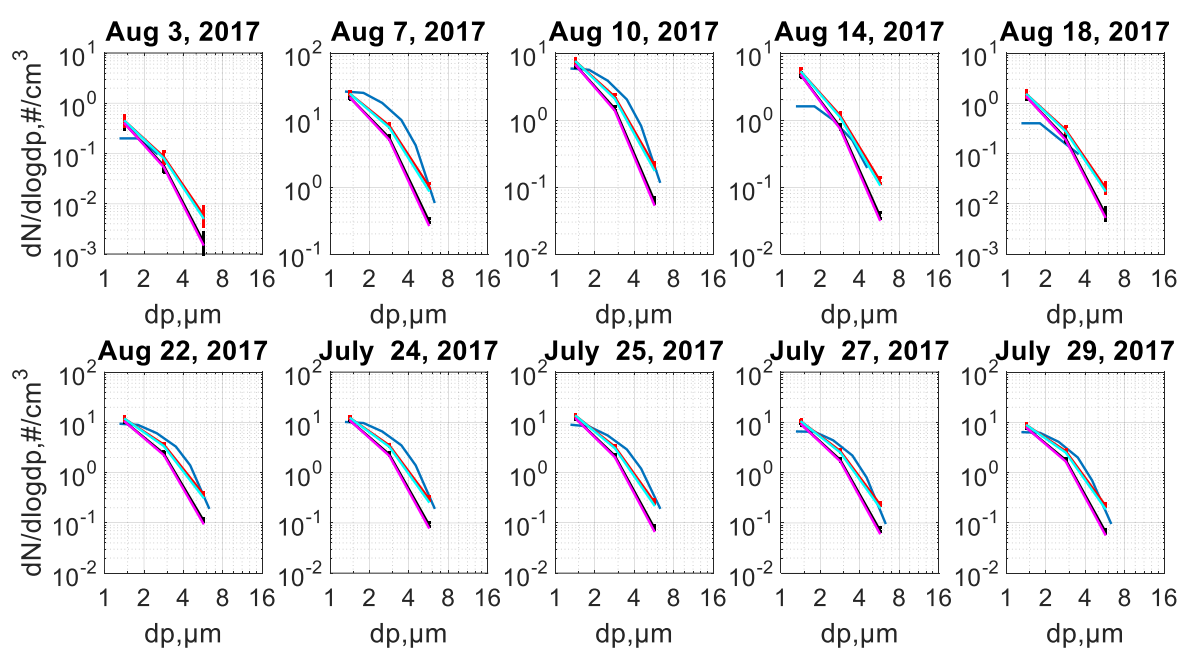

Figure S 17: Comparing number concentration calculated from deposition measurement (BSNE sampler) (Red: Wood 1981; Black: Momentum flux; Cyan: Wood 1981-PM10 inlet and Magenta: Momentum flux-PM $\mathrm{P}_{10}$ inlet) with number concentration by OPC measurement (Blue). The bars show the central $95 \%$ confidence interval of the daily variation.
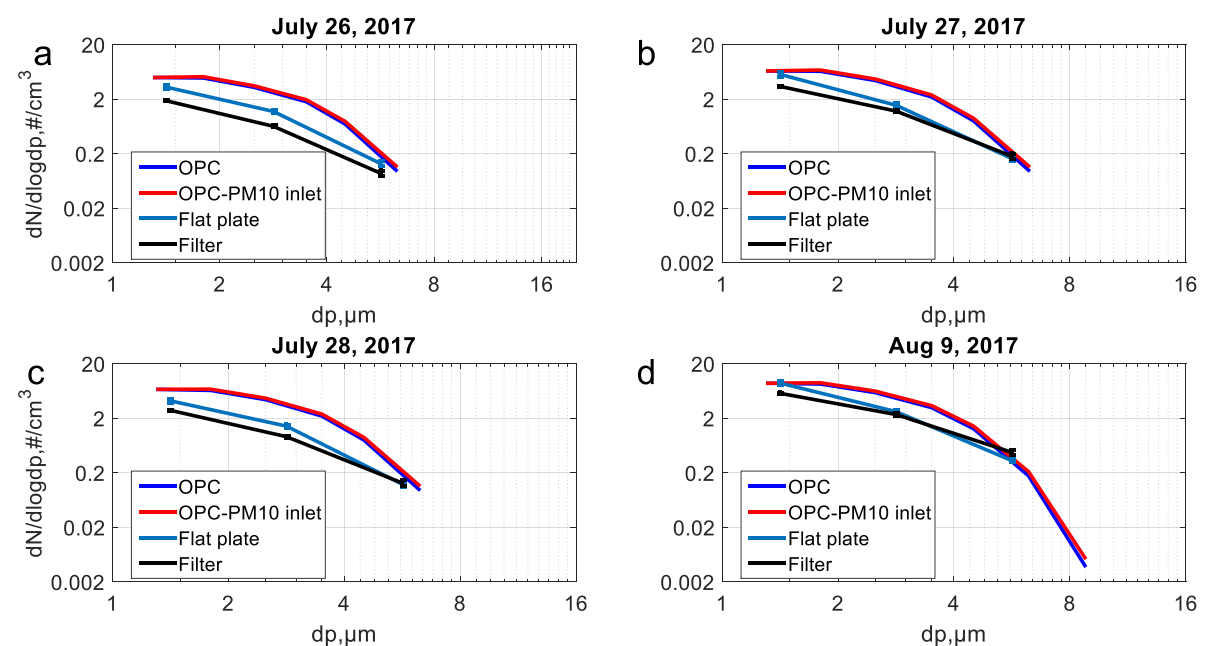

Figure S 18: Number concentration measured with Filter-sampler method, in comparison to Flat plate sampler and OPC over different measurement days (a: July 26, 2017; b: July 27, 2017; c: July 28, 
2017; d: July 29, 2017). Number concentration size distributions is obtained by converting the SEM obtained number deposition rate $\left(\left(\# /\left(\mathrm{m}^{2}\right.\right.\right.$ day $\left.)\right)$ to number concentration using different deposition velocity models. The red curve shows OPC with $\mathrm{PM}_{10}$ inlet efficiency correction. The number concentration measurement shown by filter sampler is a daily average basis. The bars show the central $95 \%$ confidence interval of the daily variation.
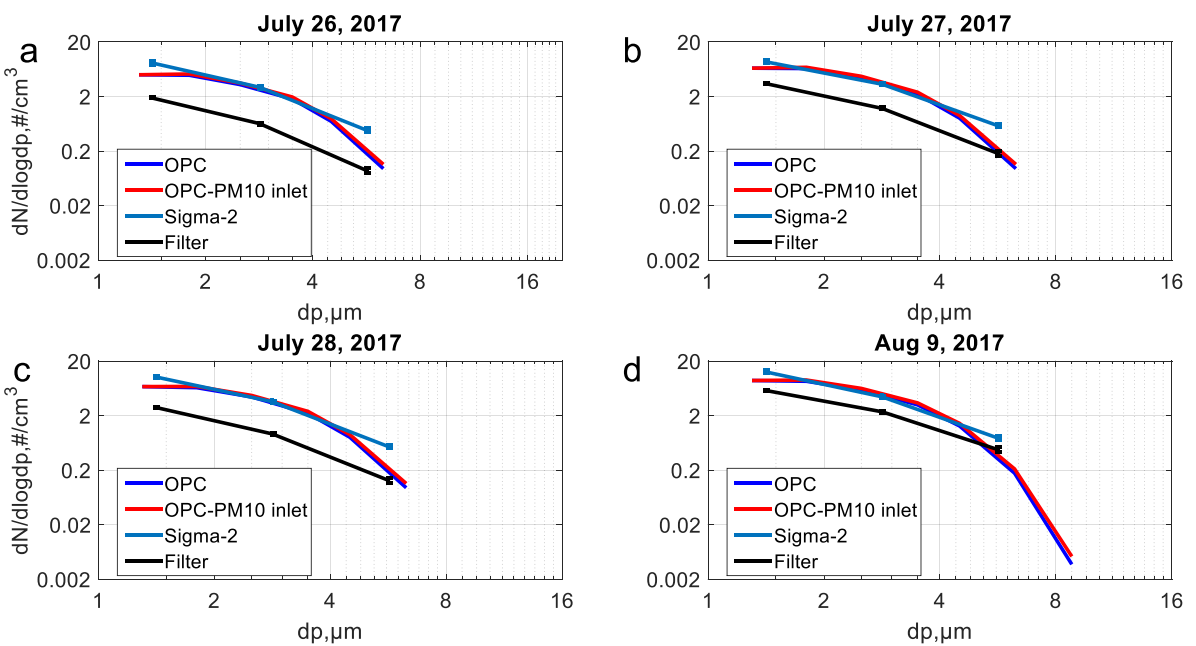

Figure S 19: Number concentration measured with Filter-sampler method, in comparison to Sigma-2 sampler and OPC over different measurement days (a: July 26, 2017; b: July 27, 2017; c: July 28, 2017; d: July 29, 2017). Number concentration size distributions is obtained by converting the SEM obtained number deposition rate $\left(\left(\# /\left(\mathrm{m}^{2}\right.\right.\right.$ day $\left.)\right)$ to number concentration using different deposition velocity models. The red curve shows OPC with $\mathrm{PM}_{10}$ inlet efficiency correction. The number concentration measurement shown by filter sampler is a daily average basis. The bars show the central $95 \%$ confidence interval of the daily variation. 

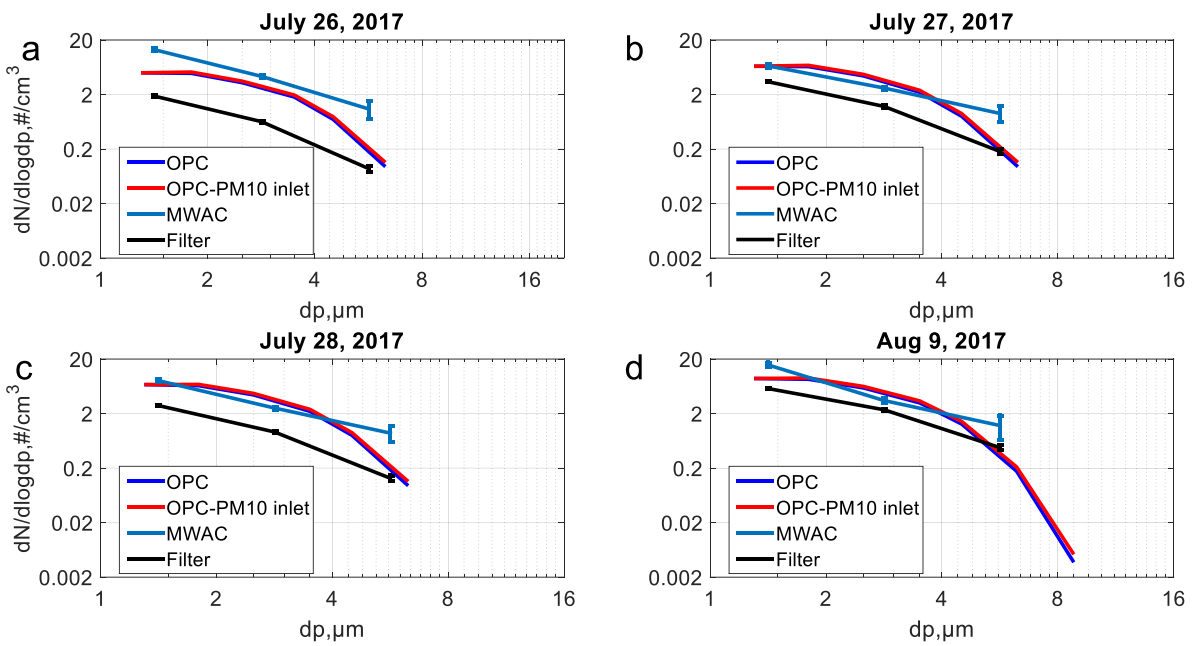

Figure S 20: Number concentration measured with Filter-sampler method, in comparison to MWAC sampler and OPC over different measurement days (a: July 26, 2017; b: July 27, 2017; c: July 28, 2017; d: July 29, 2017). Number concentration size distributions is obtained by converting the SEM obtained number deposition rate $\left(\left(\# /\left(\mathrm{m}^{2}\right.\right.\right.$ day $\left.)\right)$ to number concentration using different deposition velocity models. The red curve shows OPC with $\mathrm{PM}_{10}$ inlet efficiency correction. The number concentration measurement shown by filter sampler is a daily average basis. The bars show the central $95 \%$ confidence interval of the daily variation.

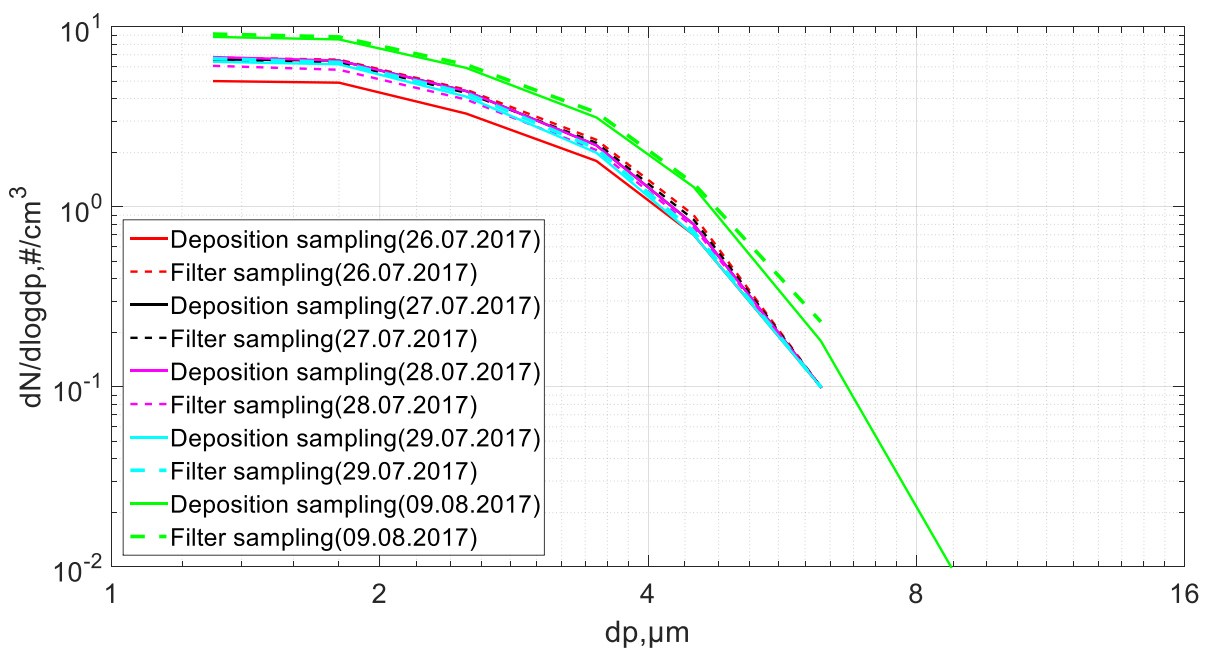


Figure S 21: An overview of the OPC measurements comparing the size distributions between the long-term (deposition) and short-term (Filter) sampling
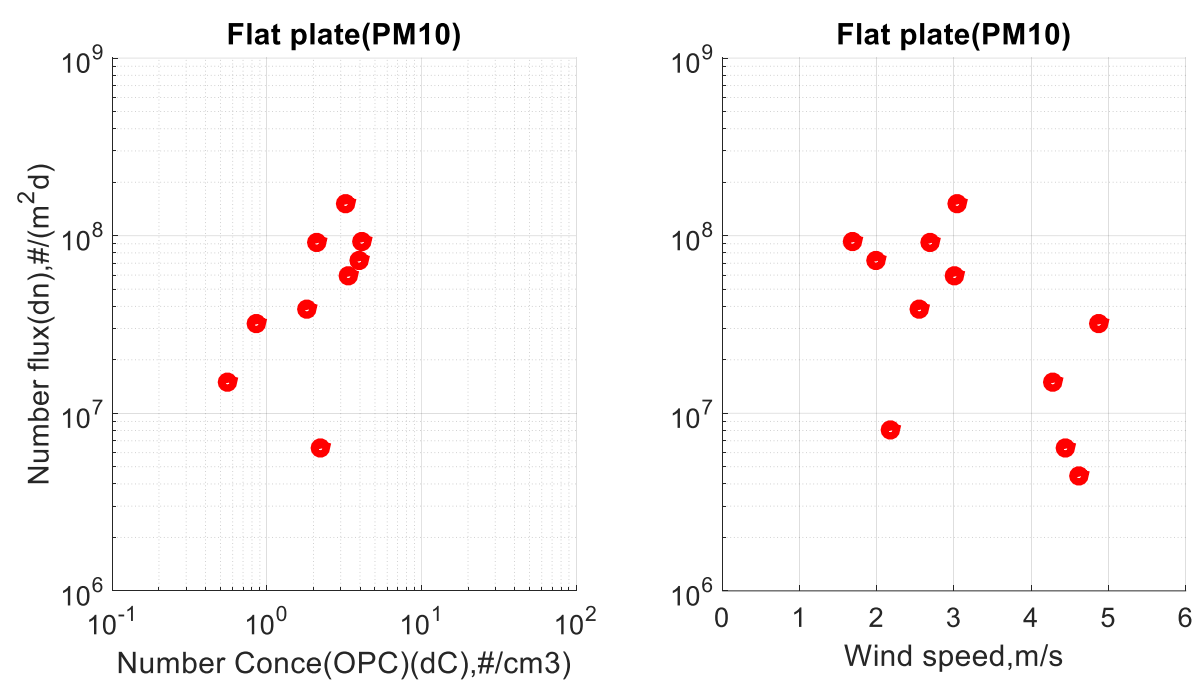

Figure S 22: Connection of deposition flux, OPC concentration, and meteorological factor (wind speed). (a) Number deposition rate of particles smaller than $10 \mu \mathrm{m}$ estimated aerodynamic diameter observed with the flat plate sampler versus number concentration of $\mathrm{PM}_{10}$ observed with the OPC. (b) Number deposition rate of particles smaller than $10 \mu \mathrm{m}$ estimated aerodynamic diameter observed with the Flat plate sampler versus the average wind speed of the collection period.
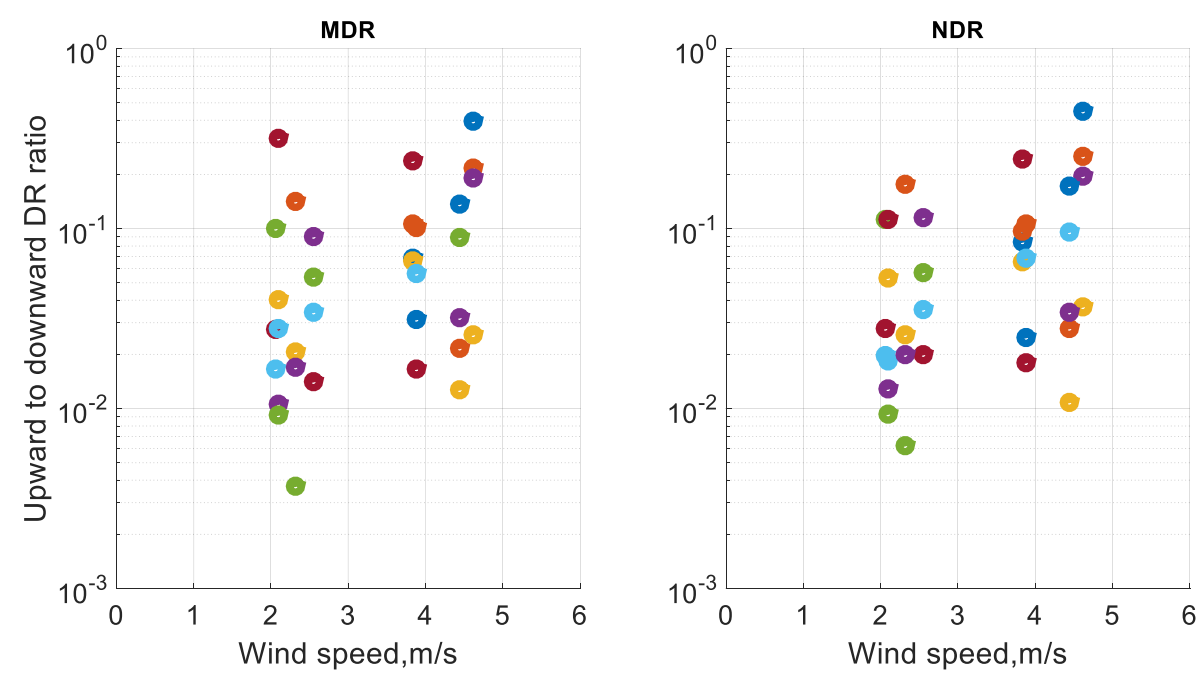
Figure S 23: Upward to downward deposition rate ratio vs wind speed. The deposition rates are measured using Flat plate sampler (with $25 \mathrm{~mm}$ stub). Different colors represent different size intervals (blue: 1-2 $\mu \mathrm{m}$; orange: 2-4 $\mu \mathrm{m}$; yellow: 4-8 $\mu \mathrm{m}$; Violet: $8-16 \mu \mathrm{m}$; green: 16-32 $\mu \mathrm{m}$; cyan: 32-64 $\mu \mathrm{m}$ ).

\section{Computational fluid dynamics (CFD) simulation}

\subsection{Detail of the sampler construction for CFD geometries}

\subsubsection{Flat plate sampler}

The bottom part of the sampler is a cylinder with a diameter of $28.9 \mathrm{~mm}$ and a height of $29 \mathrm{~mm}$ followed by another cylinder with a diameter of $40 \mathrm{~mm}$ and a height of $14 \mathrm{~mm}$. The first plate has a diameter of $127 \mathrm{~mm}$ and a thickness of $1 \mathrm{~mm}$. In the middle of the area, the deposition area is defined as a circular surface with a diameter of $12 \mathrm{~mm}$ or $25 \mathrm{~mm}$ respectively. The upper plate has the same thickness but a diameter of $203.2 \mathrm{~mm}$. Three columns hold the upper plate. The center of these columns is arranged on a diameter of $116 \mathrm{~mm}$. The diameter of the columns is $5 \mathrm{~mm}$ with a height of $16 \mathrm{~mm}$.

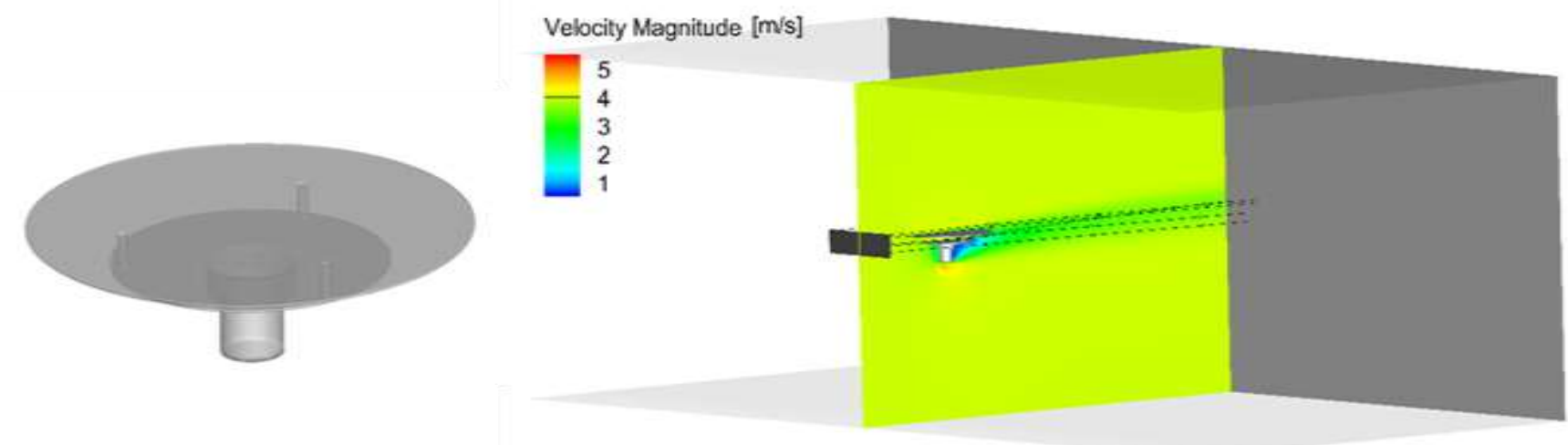

Figure S 24: Geometry of Flat plate sampler (left), CFD modeling domain and velocity magnitude, inlet velocity: $4 \mathrm{~m} / \mathrm{s}$ (right); in addition, the injection area is shown in black (width $0.2 \mathrm{~m}$, height $0.05 \mathrm{~m}$ ) along with exemplary particle trajectories.

\subsubsection{Sigma-2 sampler}

At the bottom, it consists of a mounting pole with an inner diameter of $36 \mathrm{~mm}$ and an outer diameter of $50 \mathrm{~mm}$ with a height of $32 \mathrm{~mm}$. Then follows the bottom of the sampler with a diameter of $108 \mathrm{~mm}$ and a height of $14 \mathrm{~mm}$. The wall of the geometry has an inner diameter of $104 \mathrm{~mm}$ and is $260 \mathrm{~mm}$ high. From a height of $214 \mathrm{~mm}$ the cover of the sampler starts. It has an inner diameter of $154 \mathrm{~mm}$ and an outer diameter of $158 \mathrm{~mm}$. In it, there are four openings, which start at a height of $226 \mathrm{~mm}$ and have a 
width of $40 \mathrm{~mm}$ and a height of $75 \mathrm{~mm}$. The same openings exist in the inner radius as well however turned by 90 degrees. The collector inside the geometry has a diameter of $12 \mathrm{~mm}$, a height of $13 \mathrm{~mm}$ and is positioned centrally at the bottom.

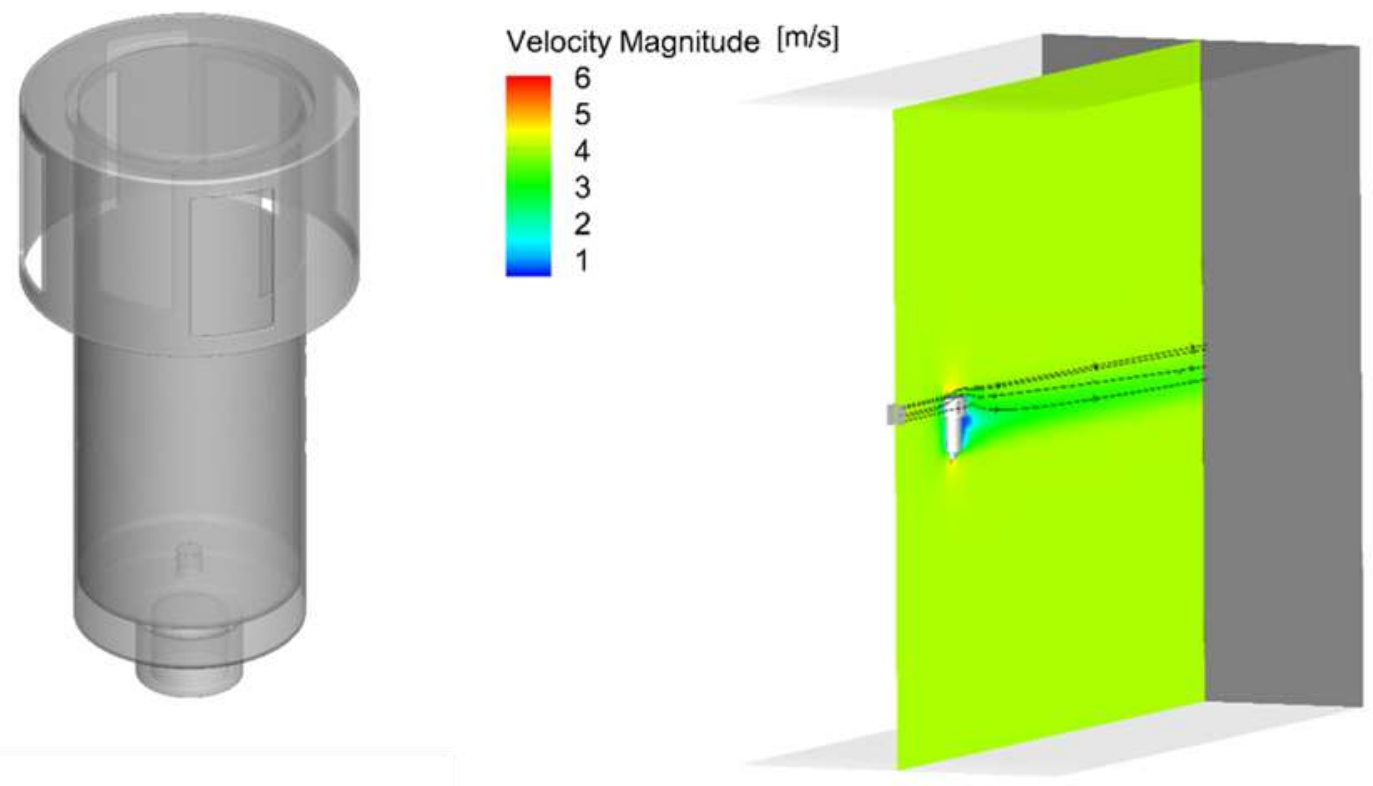

Figure S 25: Geometry of Sigma-2 sampler (left), CFD modeling domain and velocity magnitude; inlet velocity: $4 \mathrm{~m} / \mathrm{s}$ (right); in addition, the injection area is shown in black (width $0.2 \mathrm{~m}$, height $0.1 \mathrm{~m}$ ) along with exemplary particle trajectories.

\subsubsection{MWAC sampler}

The bottom diameter of the sampler is $48 \mathrm{~mm}$ with a thickness of $1.25 \mathrm{~mm}$. Afterwards the inner diameter is $45.4 \mathrm{~mm}$ up to a height of $60 \mathrm{~mm}$. The diameter then narrows semi circularly to $24 \mathrm{~mm}$. The lid of the sampler has a diameter of $40 \mathrm{~mm}$ and a height of $17 \mathrm{~mm}$.

The pipes have an inner diameter of $7.5 \mathrm{~mm}$ and a thickness of $1.25 \mathrm{~mm}$. The pipe looking into the direction of the velocity inlet is longer and ends $23 \mathrm{~mm}$ above the bottom of the sampler. The outgoing pipe starts $38 \mathrm{~mm}$ above the bottom. The collector inside the geometry has a diameter of $12 \mathrm{~mm}$, a height of $13 \mathrm{~mm}$ and is positioned centrally at the bottom. 

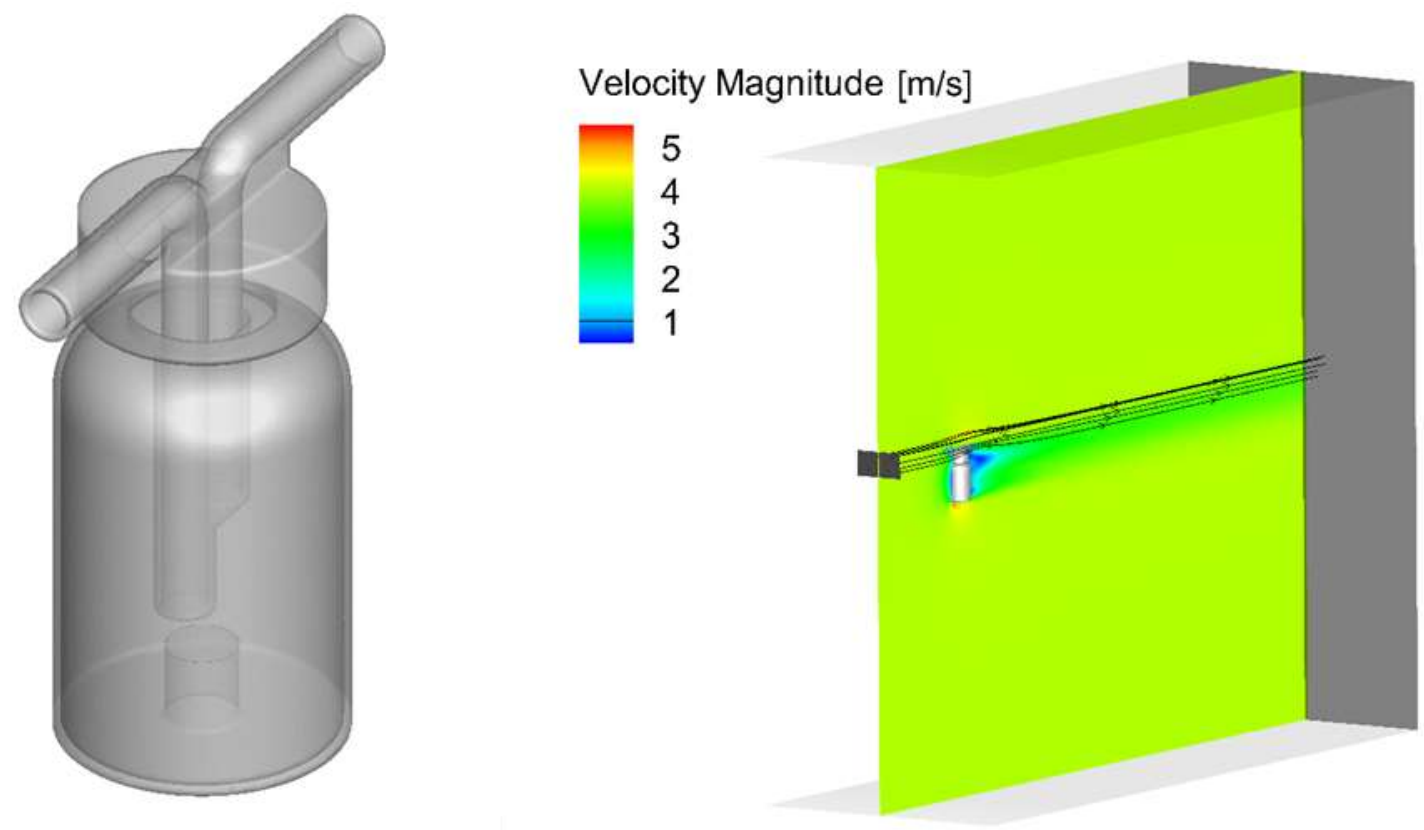

Figure S 26: Geometry of MWAC sampler (left), CFD modeling domain and velocity magnitude of MWAC sampler, inlet velocity: 4m/s (right); in addition, the injection area is shown in black (width $0.1 \mathrm{~m}$, height $0.05 \mathrm{~m}$ ) along with exemplary particle trajectories.

\subsection{Results in the cross section of the flow domain}

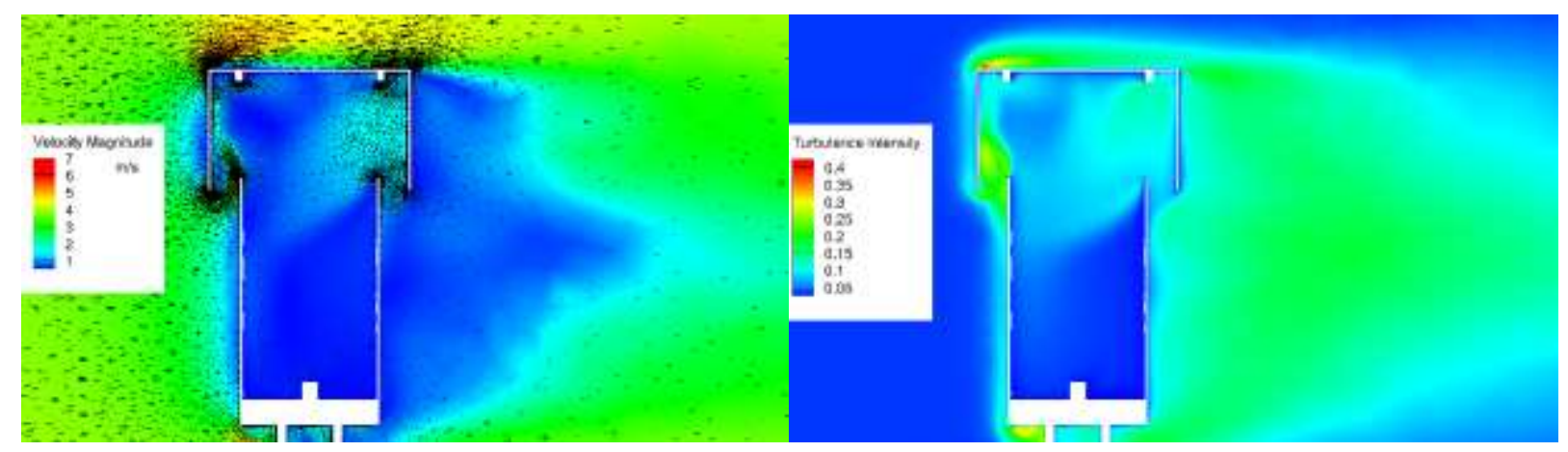

Figure S 27: Sigma-2 Sampler: Velocity magnitude and turbulence intensity at wind speed $4 \mathrm{~m} / \mathrm{s}$. 


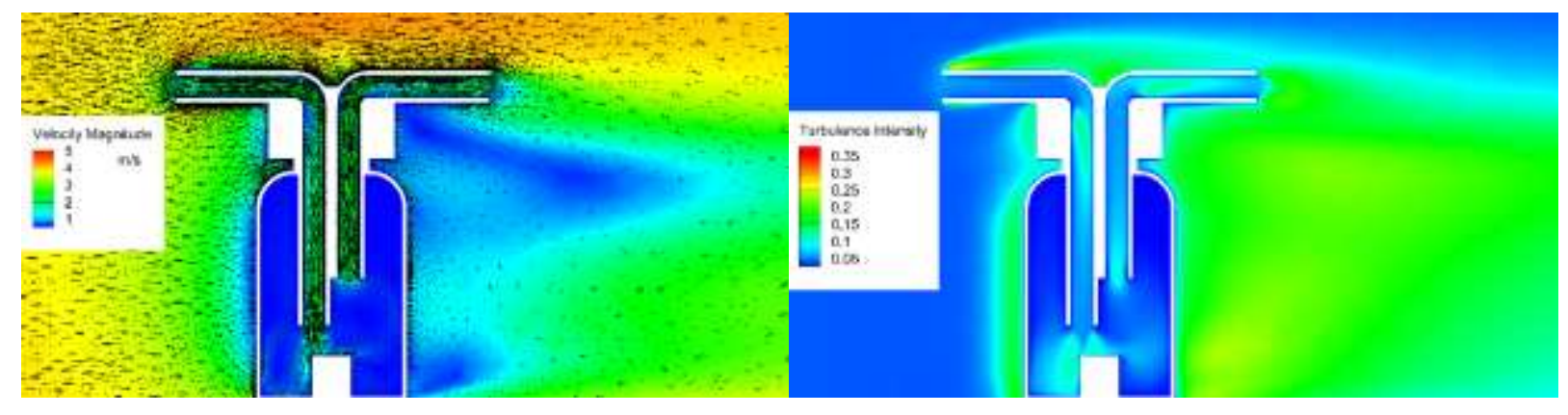

Figure S 28: MWAC Sampler: Velocity magnitude and turbulence intensity at wind speed 4 m/s.

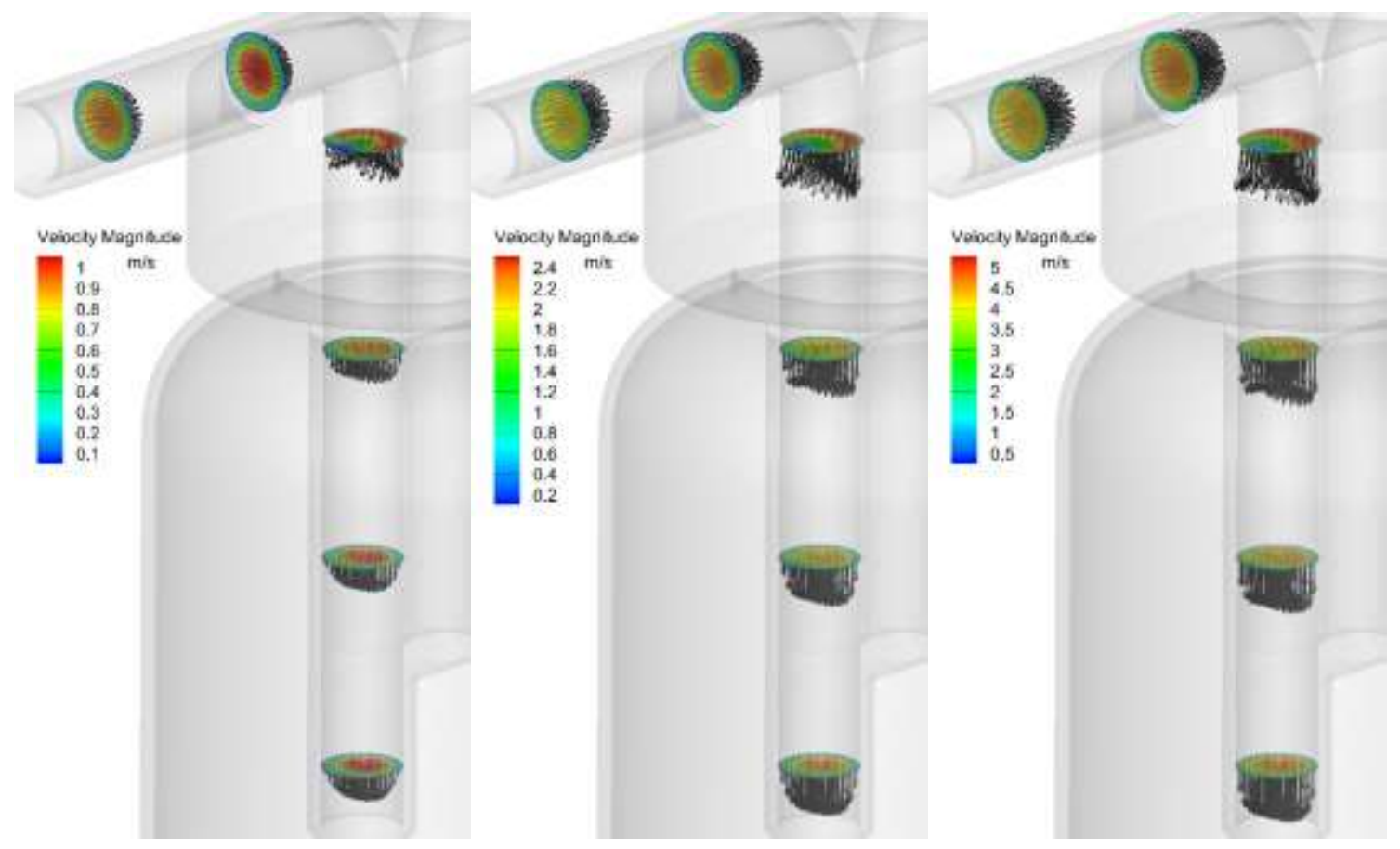

Figure S 29: Velocity vectors at 2, 4 and $8 \mathrm{~m} / \mathrm{s}$ (cross sections across and along the inlet tube). 


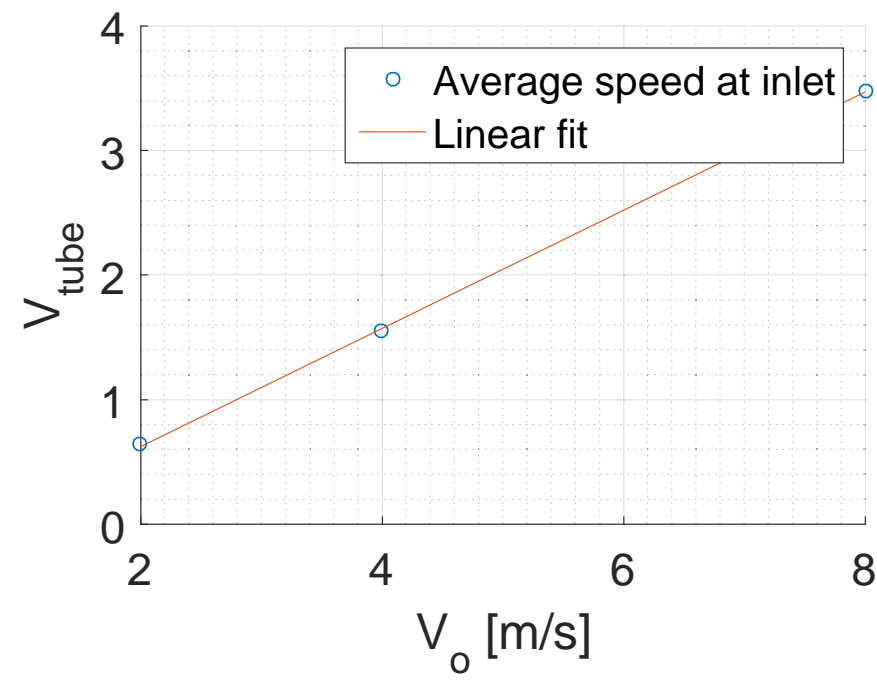

Figure S 30: Mean flow velocity ( $\left.\mathrm{V}_{\text {tube }}\right)$ in the MWAC tube as a function of the outside velocity $\left(\mathrm{V}_{\mathrm{O}}\right)$.

Fitting curve: $\mathrm{V}_{\text {tube }}=0.47 \mathrm{~V}_{0}-0.33$ for the range $2-8 \mathrm{~m} / \mathrm{s}$.

\subsection{CFD-derived deposition velocities at different wind speed}
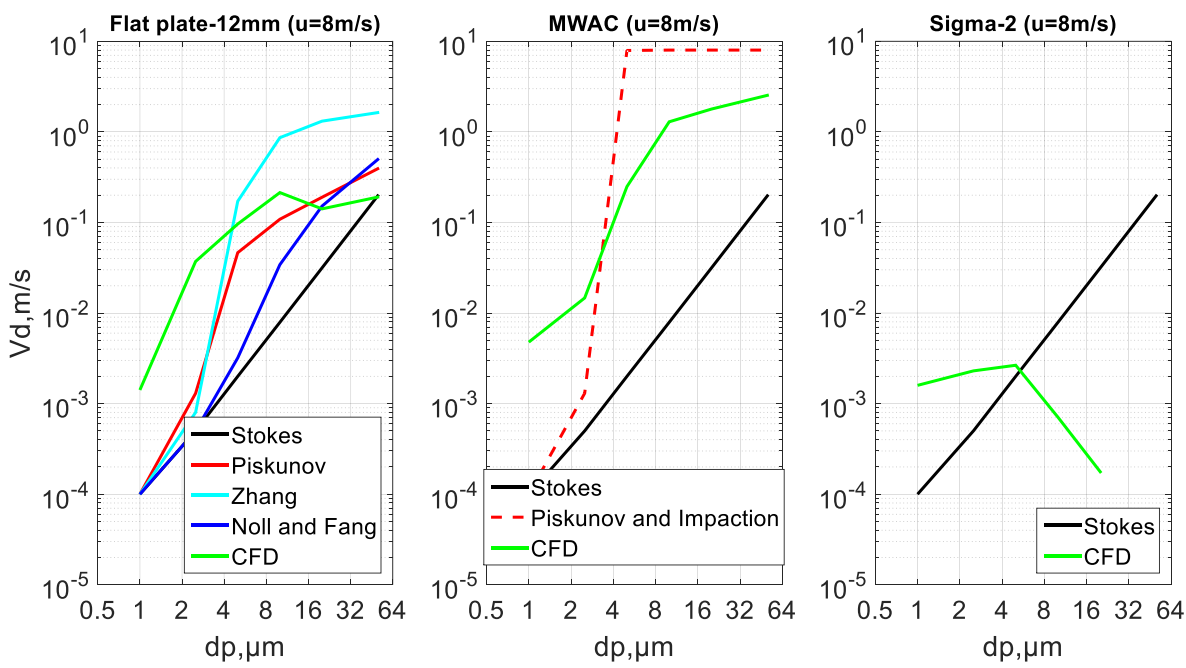

Figure S 31: Comparison of the CFD- derived deposition velocities of particles for different passive samplers to the analytical deposition velocity models used for the different samplers (at $8 \mathrm{~m} / \mathrm{s}$ wind speed). 

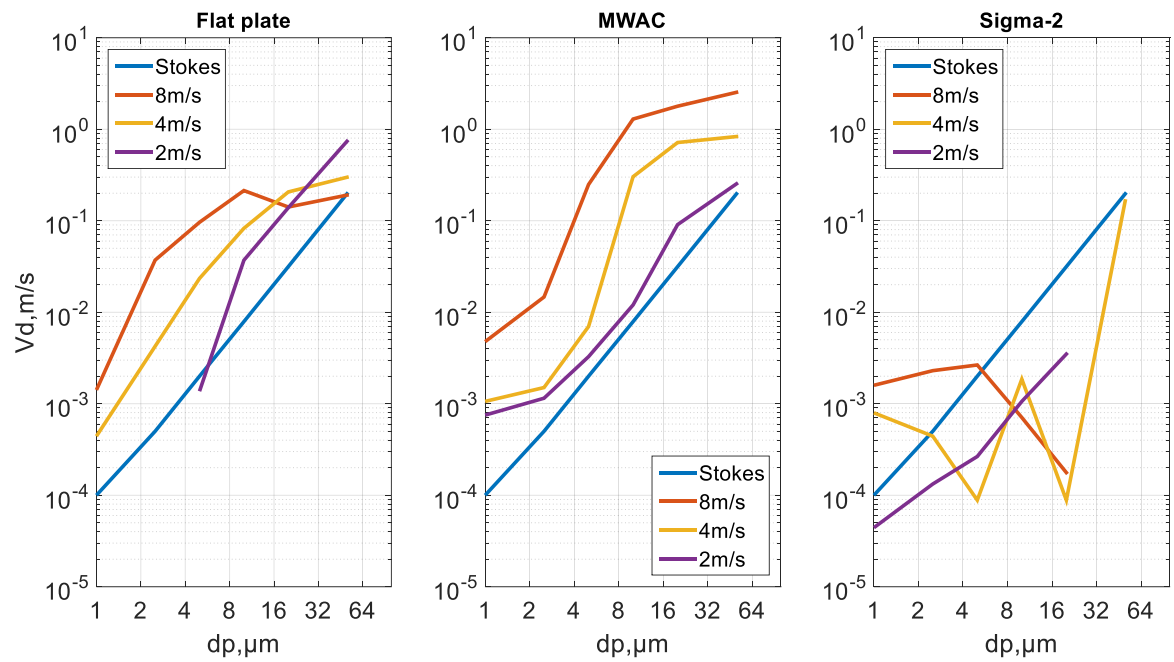

Figure S 32: Comparing the CFD-derived particle deposition velocities at different wind speed values for different samplers. 